\title{
Shift boundary material point method: an image-to-simulation workflow for solids of complex geometries undergoing large deformation
}

\author{
Chuanqi Liu ${ }^{1} \cdot$ WaiChing Sun ${ }^{1}$ (])
}

Received: 20 February 2019 / Revised: 7 April 2019 / Accepted: 17 April 2019

(c) OWZ 2019

\begin{abstract}
We introduce a mathematical framework designed to enable a simple image-to-simulation workflow for solids of complex geometries in the geometrically nonlinear regime. While the material point method is used to circumvent the mesh distortion issues commonly exhibited in Lagrangian meshes, a shifted domain technique originated from Main and Scovazzi (J Comput Phys 372:972-995, 2018) is used to represent the boundary conditions implicitly via a level set or signed distance function. Consequently, this method completely bypasses the need to generate high-quality conformal mesh to represent complex geometries and therefore allows modelers to select the space of the interpolation function without the constraints due to the geometric need. This important simplification enables us to simulate deformation of complex geometries inferred from voxel images. Verification examples on deformable body subjected to finite rotation have shown that the new shifted domain material point method is able to generate frame-indifferent results. Meanwhile, simulations using micro-CT images of a Hostun sand have demonstrated that this method is able to reproduce the quasi-brittle damage mechanisms of single grain without the excessively concentrated nodes commonly displayed in conformal meshes that represent 3D objects with local fine details.
\end{abstract}

Keywords Material point method $\cdot$ Shift domain $\cdot$ Image-based simulations $\cdot$ Nonlocal damage $\cdot$ Granular materials

\section{Introduction}

When modeling materials undergoing large deformations, finite strain models that employ Lagrangian meshes can become ineffective if severe mesh distortion occurs $[6,7$, $24,34,48,53,55,56]$. While this problem can be resolved via mesh adaptations, deriving an optimal mesh adaptation strategy is by no means an easy task. In particular, one must first have a proper remeshing criterion (e.g., configurational force or energy estimate $[33,39]$ ), a way to project internal variables $[32,54]$ and the capacity to find the new equilibrium state after mesh modifications and internal variable projections.

Mesh-free methods therefore provide an appealing alternative, which, if used properly, can enable simulations to continue even under severe large deformations. Material

\footnotetext{
WaiChing Sun

wsun@columbia.edu

1 Department of Civil Engineering and Engineering Mechanics, Columbia University, 614 SW Mudd, Mail Code: 4709 , New York, NY 10027, USA
}

point method (MPM) can be considered as one of the meshfree methods in the sense that it does not require the spatial discretization to be conformal to the geometry of the domain of the boundary value problems $[1,3,46]$. Instead, a background mesh, usually a structured mesh, is often used and the geometry of the body is represented by a set of material points that also carry all the history-dependent information (e.g., stress, internal variables and plastic deformation gradient). In each incremental time step, the background mesh is reset such that derivative operators can be built using the basis functions of the background meshes, while material points are allowed to flow across the cells of the background mesh to replicate large deformations. The facts that the current configuration of the body is not represented by a conformal mesh, but a material point set, and that the material points carry history-dependent variables make the MPM a convenient choice for modeling path-dependent materials with evolving geometries in the finite strain range $[2,25,45,47]$.

Nevertheless, modeling large deformations via the MPM is not without its own obstacles. For instance, the majority of the MPM models often employ explicit schemes for time integration. While explicit schemes may lead to a simple 
and easy implementation, the conditionally stable explicit time scheme may sometime demand a time step too small to be practical for engineering applications, such as those in geotechnical engineering [23]. In recent decades, there have been works attempting to overcome this issue via implicit formulations (e.g., Sulsky and Kaul [43], Nair and Roy [35]). However, these formulations often assume that the deformation within two consecutive incremental steps is infinitesimal and hence not suitable to capture the geomaterial nonlinearity $[8,55]$. Charlton et al. [5] developed an implicit generalized interpolation MPM (GIMP) for large deformations in which an updated Lagrangian formulation is used to update the deformation gradient and the derivatives for objects of simply geometries in one- and two-dimensional spaces. However, this method is only applicable in rectangular grids. Hence, capturing the 3D geometry and enforcing proper boundary conditions on domains of complex geometries remain major challenges.

These challenges are more profound for image-based simulations where one must introduce an efficient way to control the mesh and the material point distribution such that the degrees of freedom are invested to meet the demand for rendering the geometry of the 3D objects and sufficiently resolving the stress field to yield accurate predictions [40]. In the MPM, if we enforce the boundary conditions imposed on the true boundary with complex geometry to the nodes of a background mesh without sufficient resolution, significant errors would be introduced. Fernández-Méndez and Huerta [13] summarized different approaches for meshless methods to enforce constraints weakly via penalty, Lagrange multiplier and Nitsche's methods [36]. Recent work by Cortis et al. [8] introduced a scheme such that the essential boundary conditions can be applied on inclined planes by choosing the trial and test spaces consistent with the orientation of the inclined plane. However, this method is not applicable to curved planes where the normal vectors vary along the boundaries.

In this work, we introduce an alternative MPM formulation to simulate the behavior of $3 \mathrm{D}$ objects with complex geometries while simultaneously circumventing the difficulties of imposing essential boundary conditions. The key idea comes from the immersed and embedded boundary methods where boundary conditions are enforced implicitly in a structured mesh rather than imposed explicitly on a conformal mesh. In particular, we choose the shifted boundary method (SBM) originally proposed by Main and Scovazzi [29] for solving the Poisson and Stokes problems, the incompressible Navier-Stokes flow [30] and the hyperbolic wave problem [42], to enforce boundary conditions for the MPM. In this new shifted boundary material point method (SBMPM), the location in which the boundary condition is applied is shifted from the true boundary to a surrogate boundary. This elegant and simple treatment enables us to impose not only the
Dirichlet boundary conditions to inclined boundaries as in the recent work by Cortis et al. [8], but also arbitrary boundaries with varying normal vectors. Consequently, this integrated method provides MPM users with unprecedented capacities to capture complex geometry without excessive use of background cells solely for rendering boundaries. This freedom of designing a surrogate domain and distribution of material points without constraints due to geometric representation is particularly important for modeling path-dependent solids. In these modelings, sufficient number of degrees of freedom is required to ensure accuracy in the domain of interest where history dependent variables are evolving rapidly. To overcome the mesh sensitivity issue incurred due to softening and damage, we introduce a nonlocal damage model where the incremental constitutive update is obtained from a spatially averaged strain computed from the material points within a distance defined by the physical length scale, a technique used previously in DEM-FEM scheme (cf. Liu et al. [27]). A search algorithm is also proposed to efficiently update the set of the neighbor material points to ensure fast computation of the nonlocal strain measure. To the best knowledge of the authors, this is the first work that exploits the strength of the shifted domain method to material point models to replicate geometrically consistent material responses of solids undergoing large deformations.

The organization of the rest of the paper is as follows. We first introduce the shifted boundary material point formulation in the geometrically nonlinear regime and document the details required for constructing the tangent operator for implicit schemes (Sect. 2). Then, the material model that employs the material points to compute nonlocal strain measure is documented (Sect. 3). Due to the need to project point cloud information to a smooth field for the analysis of the simulated results, a recovery scheme based on moving least squares method is proposed in Sect. 4. This section is then followed by three numerical examples designed to both verify the formulations and demonstrate the capacity of the proposed model. A conclusion is included to summarize the major findings.

As for notations and symbols, boldfaced letters denote tensors; the symbol '. ' denotes a single contraction of adjacent indices of two tensors (e.g., $\boldsymbol{a} \cdot \boldsymbol{b}=a_{i} b_{i}$ or $\boldsymbol{c} \cdot \boldsymbol{d}=c_{i j} d_{j k}$ ); the symbol ' $:$ ' denotes a double contraction of adjacent indices of tensor of rank two or higher (e.g., $\boldsymbol{C}: \boldsymbol{\varepsilon}^{\boldsymbol{e}}=C_{i j k l} \varepsilon_{k l}^{e}$ ); the symbol ' $\otimes$ ' denotes a juxtaposition of two vectors (e.g., $\boldsymbol{a} \otimes \boldsymbol{b}=a_{i} b_{j}$ ) or two symmetric second-order tensors (e.g., $\left.(\boldsymbol{\alpha} \otimes \boldsymbol{\beta})=\alpha_{i j} \beta_{k l}\right)$.

\section{Shifted domain material point method}

In this section, we first introduce a material point method that employs the shifted boundary technique to weakly impose 
Fig. 1 True domain and surrogate domain in a background mesh

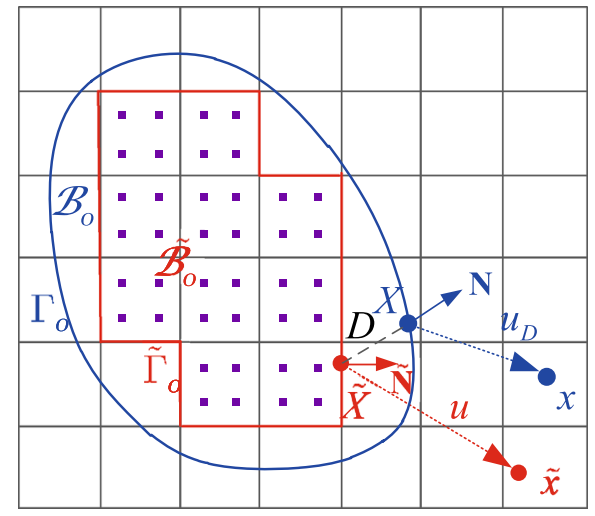

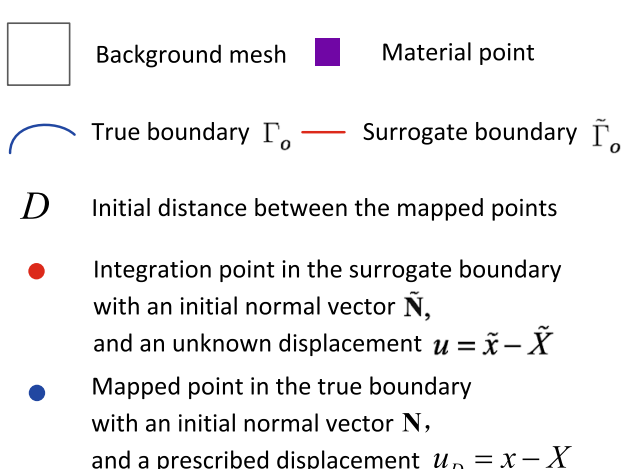

the Dirichlet (essential) boundary condition. Following this step, we briefly outline the procedure to convert the 3D voxel image into the signed distance function. This signed distance function enables us to represent the true boundary, construct a surrogate domain and set up the map between the true boundary and the surrogate boundary for the imposition of boundary conditions. To conduct quasi-static simulations, we introduce an implicit incremental algorithm and derive the tangent for the residual of the SBMPM. This section is followed by a presentation of the nonlocal isotropic damage model with a new material point neighborhood search algorithm designed for the MPM.

\subsection{Material point method with shifted boundary}

In MPM models, particles (material points) carry all physical variables via a Lagrangian description in the reference coordinates, while the derivatives are with respect to the spatial (Eulerian) coordinates. Therefore, the weak form involves integrals over the current configuration and hence suitable for an updated Lagrangian formulation. In this work, our focus is on quasi-static simulations. The quasi-static equilibrium can also be obtained via dynamic relaxation methods as demonstrated in Papadrakakis [38], Liu et al. [26,27], Wang and Sun [56]. Guilkey and Weiss [17] have provided evidence to suggest that an implicit formulation of the MPM has advantages over an explicit form. Hence, an implicit algorithm is used in this research.

In this work, the shifted domain method is used to improve the resolution and accuracy of the constraints imposed on the MPM domain of complex geometries. As demonstrated in Fig. 1, the majority of MPM formulations employ a structural mesh as background mesh. Hence, the boundary of the true domain cannot be represented properly unless the background mesh is sufficiently fine. This limitation often leads to a difficult trade-off - either employing a significantly large number of degrees of freedom on a region that may contain little valuable information for the deformation process just for the sake of representing geometry, or taking the risk of losing accuracy by using a coarse background mesh for the sake of computational efficiency.

The introduction of the shifted domain method into the MPM seamlessly resolves this constraint resolution issue such that we can strike a balance between computational efficiency and the need for geometric representation. Meanwhile, there is no need to employ a conformal mesh, as using a structured mesh enables us to directly conduct simulations on images stored as voxelized data, such as those obtained from X-ray micro-tomography [22,49-52]. Discussions on the stability and accuracy of the SBM and its applications to the fluid mechanics can be found in the original SBM work by Main and Scovazzi [29,30].

We now consider a continuum body $\mathcal{B} \subset \mathbb{R}^{n_{s d}}$ whose boundary is denoted as $\Gamma \subset \mathbb{R}^{n_{s d}-1}$, where $n_{s d}=1,2$ or 3 stands for the number of spatial dimensions. The trajectories of spatial points from the reference configuration to the current configuration are denoted as $x=\varphi(X, t)$. The undeformed continuum therefore can be denoted as $\mathcal{B}_{0}=\varphi^{-1}(\mathcal{B})$ with the boundary $\Gamma_{0}$. The deformation gradient is written as:

$\boldsymbol{F}=\frac{\partial \varphi(\boldsymbol{X}, t)}{\partial \boldsymbol{X}}=\frac{\partial \boldsymbol{x}}{\partial \boldsymbol{X}}$

The strong form of the problem in a quasi-static state reads: Find the displacement $\boldsymbol{u}: \mathcal{B} \Rightarrow \mathbb{R}^{n_{s d}}$, such that the balance of linear momentum is satisfied:

$$
\begin{aligned}
\nabla^{\boldsymbol{x}} \boldsymbol{\sigma}+\boldsymbol{b} & =\mathbf{0} \text { on } \mathcal{B}, \\
\boldsymbol{\sigma} \cdot \boldsymbol{n} & =\mathbf{0} \text { on } \Gamma_{\boldsymbol{t}}, \\
\boldsymbol{u} & =\boldsymbol{u}_{D} \text { on } \Gamma_{\boldsymbol{u}},
\end{aligned}
$$

where $\sigma$ is the Cauchy stress tensor and $\boldsymbol{b}$ is the body force. The boundary $\Gamma$ having unit normal $\boldsymbol{n}$ at $\boldsymbol{x} \in \Gamma$ holds $\Gamma=\Gamma_{t} \cup \Gamma_{u}$ and $\varnothing=\Gamma_{t} \cap \Gamma_{u}$. It should be noted that here we only consider the imposition of Dirichlet boundary conditions and we therefore assume that the traction at the natural boundary is zero. The following weak form can be 
derived by introducing a penalty parameter: find $u \in H^{1}(\mathcal{B})$ such that

$$
\begin{aligned}
g(\boldsymbol{u}, \boldsymbol{\eta})= & \int_{\mathcal{B}}\left(\nabla^{\boldsymbol{x}} \boldsymbol{\eta}: \boldsymbol{\sigma}-\boldsymbol{\eta} \cdot \boldsymbol{b}\right) \mathrm{d} v-\int_{\Gamma_{\boldsymbol{u}}} \boldsymbol{\eta} \cdot \boldsymbol{\sigma} \cdot \boldsymbol{n} \mathrm{d} s \\
& +\beta \int_{\Gamma_{\boldsymbol{u}}} \boldsymbol{\eta} \cdot\left(\boldsymbol{u}-\boldsymbol{u}_{\boldsymbol{D}}\right) \mathrm{d} s=0,
\end{aligned}
$$

for all $\eta \in H^{1}(\mathcal{B})$, where $\beta$ is a penalty parameter and $H^{1}$ denotes the Sobolev space of degree one. Note that $\eta_{\boldsymbol{x} \in \Gamma_{u}} \neq \mathbf{0}$ since the Dirichlet boundary conditions are weakly imposed. In Main and Scovazzi's method, the Dirichlet boundary condition is introduced to the weak form of governing equations by using the Nitsche's method [36]. The Nitsche's method combines the Lagrange multiplier method and the penalty method to improve the condition number and symmetry of the stiffness matrix. In practice, both the Lagrange multiplier and Nitsche's method introduce additional equations and unknowns, while the Nitsche's method may require fine-tuning of the penalty parameter to maintain accuracy and solvability. To simplify the implementation, we therefore employ the penalty method. Comparisons among different formulations will be considered in the future but are out of the scope of this study.

As shown in Fig. 1, the domain in an MPM model is discretized by a set of material points connected via a structured mesh, and hence, the true boundary cannot maintain conformal to the grid edges. We therefore shift the Dirichlet boundary conditions such that the constraint is applied to a surrogate boundary (the red line in Fig. 1) instead of the true boundary (the blue curve in Fig. 1).

Hereafter, the variables defined in the surrogate domain are denoted with a tilde. In the previous SBM, the map between the true boundary and the surrogate boundary can easily be achieved since the boundaries are fixed. Nevertheless, the deformation mapping among different configurations during the deformation process must be considered in this work. We define a map within the initial configuration:

$\boldsymbol{M}: \tilde{\Gamma}_{0} \rightarrow \Gamma_{0}, \tilde{\boldsymbol{X}} \mapsto \boldsymbol{X}$,

which maps a point $\tilde{\boldsymbol{X}} \in \tilde{\Gamma}_{0}$ in the surrogate boundary to a point $X \in \Gamma_{0}$ in the true boundary. We here construct the map by means of the closest point projection of points in $\tilde{\Gamma}_{0}$ to $\Gamma_{0}$, as illustrated in Fig. 1. The distance vector $\boldsymbol{D}$ thus is aligned with $N$, and it follows:

$D:=D N=X-\tilde{X}$,

where $D$ is the Euclidean distance between $\boldsymbol{X}$ and $\tilde{\boldsymbol{X}}$ and $\boldsymbol{N}$ is the outward-pointing unit normal at $\boldsymbol{X}=\boldsymbol{M}(\tilde{\boldsymbol{X}})$. Note that the surrogate domain is only valid if the following assumption holds,
$N \cdot \tilde{N} \geq 0$,

where $\tilde{N}$ is the outward-pointing unit normal at $\tilde{X}$. This condition $N \cdot \tilde{N} \geq 0$ means that we require that $\tilde{N}$ lies on the half-plane identified by the normal $N$, a situation that is always verified in practice as indicated in Main and Scovazzi $[29,30]$. This condition can be fulfilled by ensuring that the surrogate domain contains the topological skeleton or medial axis of the true domain [21,41]. Note that the closest point projection is not the only possible way to obtain the distance vector. The mapping $\boldsymbol{M}$ can also be constructed by solving an eikonal-type equation or explicitly computing intersections of the grid with triangulated surface as pointed out by Main and Scovazzi [29]. Readers are referred to Main and Scovazzi [29] for detailed discussion.

The spatial vector connecting the two mapped points in the current configuration becomes:

$\boldsymbol{d}=\boldsymbol{x}-\tilde{\boldsymbol{x}}=\boldsymbol{X}+\boldsymbol{u}_{D}-\tilde{\boldsymbol{X}}-\boldsymbol{u}=\boldsymbol{D}+\boldsymbol{u}_{D}-\boldsymbol{u}$,

where $\boldsymbol{u}_{D}$ is the prescribed displacement imposed on the true boundary and $\boldsymbol{u}$ is the unknown displacement imposed on the surrogate boundary. Through the map $\boldsymbol{M}$, it is possible to define the extension $\tilde{\psi}$ on $\tilde{\Gamma}$ of a function $\psi$ initially defined only on $\Gamma$, as

$\tilde{\psi}(\tilde{\boldsymbol{x}})=\psi\left(\phi\left(\boldsymbol{M}\left(\phi^{-1}(\tilde{\boldsymbol{x}})\right)\right)\right)$.

In particular, we desire to build an extension of the Dirichlet boundary condition $\boldsymbol{u}_{D}$ from the boundary $\Gamma_{\boldsymbol{u}}$ to the surrogate boundary $\tilde{\Gamma}_{\boldsymbol{u}}$, as:

$\tilde{\boldsymbol{u}}_{D}(\tilde{\boldsymbol{x}})=\boldsymbol{u}_{D}\left(\phi\left(\boldsymbol{M}\left(\phi^{-1}(\tilde{\boldsymbol{x}})\right)\right)\right)$.

Following Taylor expansion, we can further obtain the following approximations with second-order accuracy:

$$
\begin{aligned}
\left.\boldsymbol{u}(\boldsymbol{x})\right|_{\boldsymbol{x} \in \Gamma_{\boldsymbol{u}}} \approx \boldsymbol{u}(\tilde{\boldsymbol{x}})+\nabla^{\boldsymbol{x}} \boldsymbol{u} \cdot \boldsymbol{d}= & \boldsymbol{u}(\tilde{\boldsymbol{x}})+\nabla^{\boldsymbol{x}} \boldsymbol{u} \\
& \cdot\left[\boldsymbol{D}+\boldsymbol{u}_{D}-\boldsymbol{u}(\tilde{\boldsymbol{x}})\right], \\
\left.\boldsymbol{\eta}(\boldsymbol{x})\right|_{\boldsymbol{x} \in \Gamma_{\boldsymbol{u}}} \approx \boldsymbol{\eta}(\tilde{\boldsymbol{x}})+\nabla^{\boldsymbol{x}} \boldsymbol{\eta} \cdot \boldsymbol{d}= & \boldsymbol{\eta}(\tilde{\boldsymbol{x}})+\nabla^{\boldsymbol{x}} \boldsymbol{\eta} \\
& \cdot\left[\boldsymbol{D}+\boldsymbol{u}_{D}-\boldsymbol{u}(\tilde{\boldsymbol{x}})\right] .
\end{aligned}
$$

We now shift the true domain $(\mathcal{B}, \Gamma)$ to the surrogate domain $(\tilde{\mathcal{B}}, \tilde{\Gamma})$ by substituting (9), (10) and (11) into (3), and we obtain:

$$
\begin{aligned}
\tilde{g}(\boldsymbol{u}, \boldsymbol{\eta})= & \int_{\tilde{\mathcal{B}}}\left(\nabla^{\boldsymbol{x}} \boldsymbol{\eta}: \boldsymbol{\sigma}-\boldsymbol{\eta} \cdot \boldsymbol{b}\right) \mathrm{d} v-\int_{\tilde{\Gamma}_{\boldsymbol{u}}} \boldsymbol{\eta} \cdot \boldsymbol{\sigma} \cdot \tilde{\boldsymbol{n}} \mathrm{d} s \\
& +\beta \int_{\tilde{\Gamma}_{\boldsymbol{u}}}\left(\boldsymbol{\eta}+\nabla^{\boldsymbol{x}} \boldsymbol{\eta} \cdot \boldsymbol{d}\right) \cdot\left(\boldsymbol{u}+\nabla^{\boldsymbol{x}} \boldsymbol{u} \cdot \boldsymbol{d}-\tilde{\boldsymbol{u}}_{D}\right) \mathrm{d} s=0 .
\end{aligned}
$$


Before discretizing the weak form (12), we first discuss the existence and meaning of shape functions in the MPM. The most significant procedure of the MPM is to reset the background mesh at the beginning of each load step. We therefore denote the reference coordinates at the start of $n$th load step as $X_{n}=\varphi\left(X, t_{n}\right)$. The weighting function at the node $I$ therefore is only meaningful with the current coordinates, $N_{I}=N_{I}(\boldsymbol{x})$, or the updated reference coordinates, $N_{I}=N_{I}\left(\boldsymbol{X}_{n}\right)$. We here adopt the shape functions as $N_{I}=N_{I}\left(\boldsymbol{X}_{n}\right)$ and approximate the displacements as:

$\boldsymbol{u}=N_{I} \boldsymbol{u}_{I}, \quad \boldsymbol{\eta}=N_{I} \boldsymbol{\eta}_{I}$,

where the repeated subscript $I$ satisfies the Einstein summation convention. As a result, in our MPM formulation, the current deformation gradient can be split into two terms, the deformation gradient at $t=t_{n}$ and the incremental change, i.e.,

$$
\begin{aligned}
\boldsymbol{F} & =\frac{\partial \boldsymbol{x}}{\partial \boldsymbol{X}}=\frac{\partial\left(\boldsymbol{X}_{n}+\Delta \boldsymbol{u}\right)}{\partial \boldsymbol{X}}=\frac{\partial \boldsymbol{X}_{n}}{\partial \boldsymbol{X}}+\frac{\partial \Delta \boldsymbol{u}}{\partial \boldsymbol{X}_{n}} \frac{\partial \boldsymbol{X}_{n}}{\partial \boldsymbol{X}} \\
& =\left(\boldsymbol{I}+\frac{\partial \Delta \boldsymbol{u}}{\partial \boldsymbol{X}_{n}}\right) \frac{\partial \boldsymbol{X}_{n}}{\partial \boldsymbol{X}}=\boldsymbol{F}_{\Delta} \boldsymbol{F}_{n}
\end{aligned}
$$

where $\boldsymbol{F}_{\Delta}$ is the incremental deformation gradient and can be expressed as:

$\boldsymbol{F}_{\Delta}=\boldsymbol{I}+\frac{\partial \Delta \boldsymbol{u}}{\partial \boldsymbol{X}_{n}}=\frac{\partial \boldsymbol{x}}{\partial \boldsymbol{X}_{n}}$

The gradient of shape function with regard to the current coordinates therefore is:

$\frac{\partial N_{I}\left(\boldsymbol{X}_{n}\right)}{\partial \boldsymbol{x}}=\frac{\partial N_{I}\left(\boldsymbol{X}_{n}\right)}{\partial \boldsymbol{X}_{n}} \frac{\partial \boldsymbol{X}_{n}}{\partial \boldsymbol{x}}=\frac{\partial N_{I}\left(\boldsymbol{X}_{n}\right)}{\partial \boldsymbol{X}_{n}} \boldsymbol{F}_{\Delta}^{-1}$.

\subsection{Generating geometry via signed distance function}

As discussed above, the geometry of the true domain is required for the construction of the surrogate domain and the corresponding map. The most straightforward quantity is the signed distance function for a body. The signed distance function is a mapping that takes a position vector $\boldsymbol{x}$ and returns a scalar value $\phi(\boldsymbol{x})$. The sign of this scalar indicates whether the point at $\boldsymbol{x}$ is inside or outside a body and the absolute value of $\phi(x)$ is the Euclidean distance measured by $\|\cdot\|$, i.e.,

$$
\phi(\boldsymbol{x})=\operatorname{sgn}(\boldsymbol{x}) \min _{\overline{\boldsymbol{x}} \in \Gamma}\|\boldsymbol{x}-\overline{\boldsymbol{x}}\|,
$$

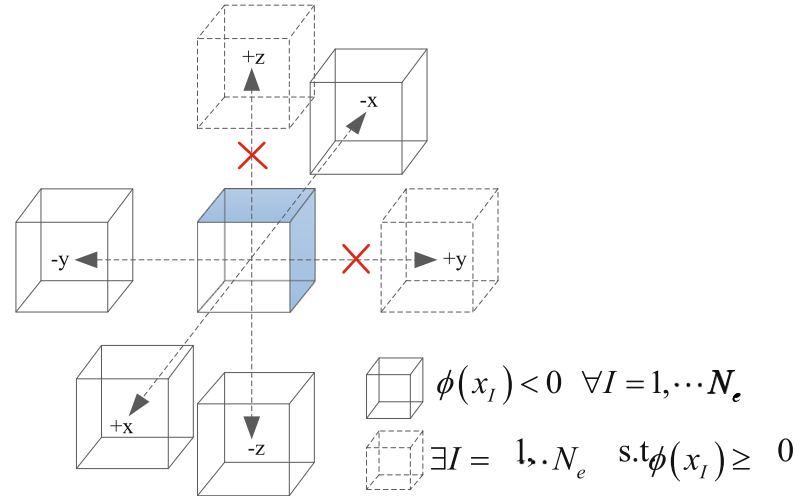

Fig. 2 Determining the surrogate surfaces (in blue color). (Color figure online)

where sgn $(\boldsymbol{x})$ is the sign function and defined as:

$\operatorname{sgn}(\boldsymbol{x})=\left\{\begin{array}{rl}1 & \boldsymbol{x} \in \mathcal{B} \\ -1 & \text { otherwise }\end{array}\right.$

In this work, we use the convention where $\phi(x)<0$ indicates the exterior and $\phi(x)>0$ the interior of the true domain. On the boundary, $\phi(x)=0$. Once we obtain the field of the signed distance function, the true boundary can be reconstructed by a set of triangles by using marching cube algorithms [28]. We then set up the map between the true boundary and a surrogate boundary by connecting integration points on the surrogate surface to their closest points on the triangles representing the true boundary. Note that for simple shapes, such as sphere or cube, we can set up the map analytically.

The field of the signed distance function can also be employed to determine the surrogate domain. We have mentioned that the surrogate domain should be inside the true domain in the SBM. As shown in Fig. 2, one element is labeled as an interior element if $\phi\left(\boldsymbol{x}_{I}\right)<0$ for $\forall I=$ $1, \ldots, N_{e}$, where $N_{e}$ is the number of nodes per element. Otherwise, the element would be labeled as an exterior element when $\exists I=1, \ldots, N_{e}$, such that $\phi\left(\boldsymbol{x}_{I}\right) \geq 0$. One cube has six faces corresponding to its six neighboring elements. If the cube is an interior element and one of its neighboring elements is an exterior element, the connecting face between these two elements would be part of the boundary of the surrogate domain, as shown in the blue faces in Fig. 2. After looping all elements, we can straightforwardly generate a surrogate boundary and the corresponding surrogate domain consisting of squares and cubes, respectively.

\subsection{Linearization for the implicit scheme}

To obtain incremental update of the MPM solution implicitly, we perform the consistent linearization of the weak form (12) 
in the current configuration at $\boldsymbol{u}=\boldsymbol{u}^{*}$ and the consistent linearization reads

$L[\tilde{g}]_{\boldsymbol{u}=\boldsymbol{u}^{*}}=\tilde{g}\left(\boldsymbol{u}^{*}, \boldsymbol{\eta}\right)+D \tilde{g}\left(\boldsymbol{u}^{*}, \boldsymbol{\eta}\right) \cdot \delta \boldsymbol{u}$,

where

$$
\begin{aligned}
& \tilde{g}\left(\boldsymbol{u}^{*}, \boldsymbol{\eta}\right)=\underbrace{\int_{\tilde{\mathcal{B}}} \nabla^{\boldsymbol{x}} \boldsymbol{\eta}: \boldsymbol{\sigma}^{*} \mathrm{~d} v}_{\eta_{I} f_{\mathrm{int} 1}}-\underbrace{\int_{\tilde{\mathcal{B}}} \boldsymbol{\eta} \cdot \boldsymbol{b} \mathrm{d} v}_{\eta_{I} \boldsymbol{f}_{\mathrm{ext} 1}}-\underbrace{\int_{\tilde{\Gamma}_{u}} \boldsymbol{\eta} \cdot \boldsymbol{\sigma}^{*} \cdot \tilde{\boldsymbol{n}} \mathrm{d} s}_{\eta_{I} f_{\mathrm{int} 2}} \\
& +\beta \underbrace{\int_{\tilde{\Gamma}_{u}} \boldsymbol{\eta} \cdot \boldsymbol{u}^{*} \mathrm{~d} s}_{\boldsymbol{\eta}_{I} \boldsymbol{f}_{\mathrm{fin} 13}}+\beta \underbrace{\int_{\tilde{\Gamma}_{u}} \boldsymbol{\eta} \cdot \nabla^{\boldsymbol{x}} \boldsymbol{u}^{*} \cdot \boldsymbol{d}^{*} \mathrm{~d} s}_{\eta_{I} \boldsymbol{f}_{\mathrm{int} 4}}-\beta \underbrace{\int_{\tilde{\Gamma}_{u}} \boldsymbol{\eta} \cdot \tilde{\boldsymbol{u}}_{D} \mathrm{~d} s}_{\boldsymbol{\eta}_{I} \boldsymbol{f}_{\mathrm{ext2}}} \\
& +\beta \underbrace{\int_{\tilde{\Gamma}_{u}} \nabla^{\boldsymbol{x}} \boldsymbol{\eta} \cdot \boldsymbol{d}^{*} \cdot \boldsymbol{u}^{*} \mathrm{~d} s}_{\eta_{I} f_{\mathrm{int} 5}}+\beta \underbrace{\int_{\tilde{\Gamma}_{u}} \nabla^{\boldsymbol{x}} \boldsymbol{\eta} \cdot \boldsymbol{d}^{*} \cdot \nabla^{\boldsymbol{x}} \boldsymbol{u}^{*} \cdot \boldsymbol{d}^{*} \mathrm{~d} s}_{\eta_{I} \boldsymbol{f}_{\mathrm{int} 6}} \\
& -\beta \underbrace{\int_{\tilde{\Gamma}_{u}} \nabla^{x} \eta \cdot \boldsymbol{d}^{*} \cdot \tilde{\boldsymbol{u}}_{D} \mathrm{~d} s}_{\eta_{I} f_{\mathrm{int} 7}}
\end{aligned}
$$

and

$$
\begin{aligned}
& D \tilde{g}\left(\boldsymbol{u}^{*}, \boldsymbol{\eta}\right) \cdot \delta \boldsymbol{u} \\
& =\underbrace{\int_{\tilde{\mathcal{B}}} \nabla^{\boldsymbol{x}} \boldsymbol{\eta}: \boldsymbol{a}: \nabla^{\boldsymbol{x}} \delta \boldsymbol{u} \mathrm{d} v}_{\eta_{I} \boldsymbol{K}_{1} \delta \boldsymbol{u}_{I}}-\underbrace{\int_{\tilde{\Gamma}_{u}} \boldsymbol{\eta} \cdot \boldsymbol{a}: \nabla^{\boldsymbol{x}} \delta \boldsymbol{u} \cdot \tilde{\boldsymbol{n}} \mathrm{d} s}_{\boldsymbol{\eta}_{I} \boldsymbol{K}_{2} \delta \boldsymbol{u}_{I}}+\beta \underbrace{\int_{\tilde{\Gamma}_{\boldsymbol{u}}} \boldsymbol{\eta} \cdot \delta \boldsymbol{u} \mathrm{d} s}_{\boldsymbol{\eta}_{I} \boldsymbol{K}_{3} \delta \boldsymbol{u}_{I}} \\
& +\beta \underbrace{\int_{\tilde{\Gamma}_{u}} \boldsymbol{\eta} \cdot\left(\nabla^{\boldsymbol{x}} \delta \boldsymbol{u} \cdot \boldsymbol{d}^{*}-\nabla^{\boldsymbol{x}} \boldsymbol{u}^{*} \cdot \delta \boldsymbol{u}\right) \mathrm{d} s}_{\eta_{I} \boldsymbol{K}_{4} \delta \boldsymbol{u}_{I}} \\
& +\beta \underbrace{\int_{\tilde{\Gamma}_{u}} \nabla^{\boldsymbol{x}} \boldsymbol{\eta} \cdot\left(\boldsymbol{d}^{*} \cdot \delta \boldsymbol{u}-\delta \boldsymbol{u} \cdot \boldsymbol{u}^{*}\right) \mathrm{d} s}_{\eta_{I} \boldsymbol{K}_{5} \delta \boldsymbol{u}_{I}} \\
& +\beta \underbrace{\int_{\tilde{\Gamma}_{u}} \nabla^{\boldsymbol{x}} \boldsymbol{\eta} \cdot\left(\boldsymbol{d}^{*} \cdot \nabla^{\boldsymbol{x}} \delta \boldsymbol{u} \cdot \boldsymbol{d}^{*}-\boldsymbol{d}^{*} \cdot \nabla^{\boldsymbol{x}} \boldsymbol{u}^{*} \cdot \delta \boldsymbol{u}-\delta \boldsymbol{u} \cdot \nabla^{\boldsymbol{x}} \boldsymbol{u}^{*} \cdot \boldsymbol{d}^{*}\right) \mathrm{d} s}_{\eta_{I} \boldsymbol{K}_{6} \delta \boldsymbol{u}_{I}} \\
& +\beta \underbrace{\int_{\tilde{\Gamma}_{u}} \nabla^{\boldsymbol{x}} \boldsymbol{\eta} \cdot \delta \boldsymbol{u} \cdot \tilde{\boldsymbol{u}}_{D} \mathrm{~d} s}_{\eta_{I} \boldsymbol{K}_{7} \delta \boldsymbol{u}_{I}}
\end{aligned}
$$

In the above derivation of the tangent, we linearized all the terms varying with $\boldsymbol{u}$, i.e., $\boldsymbol{\sigma}=\boldsymbol{\sigma}(\boldsymbol{u}), \boldsymbol{d}=\boldsymbol{d}(\boldsymbol{u})$ and $\boldsymbol{u}=\boldsymbol{u}$. For the sake of convenience, the terms involving $\sigma$ (i.e., $D \boldsymbol{f}_{\text {int } 1} \cdot \delta \boldsymbol{u}$ and $\left.D \boldsymbol{f}_{\text {int2 }} \cdot \delta \boldsymbol{u}\right)$, are derived by a pullback into the reference configuration. The procedures are standard and shown in "Appendix". For the derivations of the other terms, we need to consider $\boldsymbol{D}$ and $\boldsymbol{u}_{D}$ in (7) are constant vectors and therefore directly derivate the terms by using $\boldsymbol{d}^{*}=\boldsymbol{d}\left(\boldsymbol{u}^{*}\right)$ and $\delta \boldsymbol{d}=-\delta \boldsymbol{u}$. It should be mentioned that the $\boldsymbol{a}$ is termed gener- ally the spatial tangent modulus and defined by the Cartesian components as

$a_{i j k l}=\frac{1}{J} A_{i m k n} F_{j m} F_{l n}=\frac{1}{J} \frac{\partial \tau_{i j}}{\partial F_{k m}} F_{l m}-\sigma_{i l} \delta_{j k}$,

where $J=\operatorname{det} \boldsymbol{F}$ is the Jacobian, $\boldsymbol{A} \equiv \frac{\partial \boldsymbol{P}}{\partial \boldsymbol{F}}$ is the material tangent modulus, $\boldsymbol{P}$ is the first Piola-Kirchhoff stress tensor, $\boldsymbol{F}$ is the deformation gradient, $\boldsymbol{\tau}$ is the Kirchhoff stress and $\delta$ is the Kronecker delta tensor. A more specific expression of $\boldsymbol{a}$ would be given after introducing the constitutive law.

In terms of the matrix form, we consider a linear cubic element with eight nodes for the background mesh and introduce the following notations for clarity.

1. A second-order tensor $\boldsymbol{c}$ with $3 \times 3$ elements is either referred to a matrix as $\overline{\bar{c}}$ or a vector as $\bar{c}$ and their correlation is $\overline{\bar{c}}_{i j}=\bar{c}_{k}$ where $k=(i-1) \times 3+j$ with $i, j=1,2,3$ and $k=1, \ldots, 9$.

2. $\boldsymbol{u}^{*}$ is a vector with length of 3 , and $\boldsymbol{u}^{* \text { node }}$ is the vector of nodal displacement with length of $3 \times 8=24$. We thus can obtain $\boldsymbol{u}^{*}$ and $\nabla^{\boldsymbol{x}} \boldsymbol{u}^{*}$ within the element as follows:

$$
\boldsymbol{u}^{*}=\boldsymbol{N} \boldsymbol{u}^{* \text { node }}, \quad \nabla^{\boldsymbol{x}} \boldsymbol{u}^{*}=\overline{\overline{\boldsymbol{G} \boldsymbol{u}^{* \text { node }}}} \text { or } \overline{\boldsymbol{G} \boldsymbol{u}^{* \text { node }}},
$$

where $\boldsymbol{N}$ is the matrix form of shape function and $\boldsymbol{G}$ is the full gradient operator. Note that here elements in $\boldsymbol{G}$ are the derivatives of the shape functions $N_{I}\left(\boldsymbol{X}_{n}\right)$ with respect to the spatial coordinates $\boldsymbol{x}$.

3. We introduce matrices $\boldsymbol{R}^{d}$ and $\boldsymbol{r}^{d}$ with size of $3 \times 9$ for each to ensure:

$$
\overline{\overline{\boldsymbol{c}}} \cdot \boldsymbol{d}^{*}=\boldsymbol{R}^{d} \overline{\boldsymbol{c}}=\left(\overline{\boldsymbol{c}}^{T}\left(\boldsymbol{R}^{d}\right)^{T}\right)^{T} \text { and }\left(\boldsymbol{d}^{*}\right)^{T} \overline{\overline{\boldsymbol{c}}}=\overline{\boldsymbol{c}}^{T}\left(\boldsymbol{r}^{d}\right)^{T}
$$

Similarly, we can define $\boldsymbol{R}^{u}, \boldsymbol{r}^{u}, \boldsymbol{R}^{n}, \boldsymbol{r}^{u d}$ and $\boldsymbol{r}^{u D}$ to ensure that $\overline{\bar{c}} \cdot \boldsymbol{u}^{*}=\boldsymbol{R}^{u} \overline{\boldsymbol{c}}=\left(\overline{\boldsymbol{c}}^{T}\left(\boldsymbol{R}^{u}\right)^{T}\right)^{T},\left(\boldsymbol{u}^{*}\right)^{T} \overline{\bar{c}}=$ $\overline{\boldsymbol{c}}^{T}\left(\boldsymbol{r}^{u}\right)^{T}, \overline{\overline{\boldsymbol{c}}} \cdot \tilde{\boldsymbol{n}}=\boldsymbol{R}^{n} \overline{\boldsymbol{c}},\left(\nabla^{\boldsymbol{x}} \boldsymbol{u}^{*} \cdot \boldsymbol{d}^{*}\right)^{T} \overline{\overline{\boldsymbol{c}}}=\overline{\boldsymbol{c}}^{T}\left(\boldsymbol{r}^{u d}\right)^{T}$ and $\left(\tilde{\boldsymbol{u}}_{D}\right)^{T} \cdot \overline{\overline{\boldsymbol{c}}}=\overline{\boldsymbol{c}}^{T}\left(\boldsymbol{r}^{u D}\right)^{T}$, respectively.

Each term in (20) and (21) thus can be expressed as:

$$
\left\{\begin{array}{l}
\boldsymbol{f}_{\mathrm{int} 1}=\int_{\tilde{\mathcal{B}}} \boldsymbol{G}^{T} \overline{\boldsymbol{\sigma}}^{*} \mathrm{~d} v, \quad \boldsymbol{f}_{\mathrm{int} 2}=\int_{\tilde{\Gamma}_{u}} \boldsymbol{N}^{T} \boldsymbol{R}^{n} \overline{\boldsymbol{\sigma}}^{*} \mathrm{~d} s, \quad \boldsymbol{f}_{\mathrm{int} 3}=\int_{\tilde{\Gamma}_{u}} \boldsymbol{N}^{T} \boldsymbol{N} \boldsymbol{u}^{* \text { node }} \mathrm{d} s, \\
\boldsymbol{f}_{\mathrm{int} 4}=\int_{\tilde{\Gamma}_{u}} \boldsymbol{N}^{T} \boldsymbol{R}^{d} \overline{\boldsymbol{G} \boldsymbol{u}^{* \mathrm{node}}} \mathrm{d} s, \quad \boldsymbol{f}_{\mathrm{int} 5}=\int_{\tilde{\Gamma}_{u}} \boldsymbol{G}^{T}\left(\boldsymbol{R}^{d}\right)^{T} \boldsymbol{N} \boldsymbol{u}^{* \text { node }} \mathrm{d} s, \\
\boldsymbol{f}_{\mathrm{int} 6}=\int_{\tilde{\Gamma}_{u}} \boldsymbol{G}^{T}\left(\boldsymbol{R}^{d}\right)^{T} \boldsymbol{R}^{d} \overline{\boldsymbol{G} \boldsymbol{u}^{* \mathrm{node}}} \mathrm{d} s, \quad \boldsymbol{f}_{\mathrm{int} 7}=\int_{\tilde{\Gamma}_{u}} \boldsymbol{G}^{T}\left(\boldsymbol{R}^{d}\right)^{T} \tilde{\boldsymbol{u}}_{D} \mathrm{~d} s, \\
\boldsymbol{f}_{\mathrm{ext} 1}=\int_{\tilde{\mathcal{B}}} \boldsymbol{N}^{T} \boldsymbol{b} \mathrm{d} v, \quad \boldsymbol{f}_{\mathrm{ext} 2}=\int_{\tilde{\Gamma}_{u}} \boldsymbol{N}^{T} \tilde{\boldsymbol{u}}_{D} \mathrm{~d} s,
\end{array}\right.
$$


and

$$
\left\{\begin{array}{l}
\boldsymbol{K}_{1}=\int_{\tilde{\mathcal{B}}} \boldsymbol{G}^{T} \boldsymbol{a} \boldsymbol{G} \mathrm{d} v, \quad \boldsymbol{K}_{2}=\int_{\tilde{\Gamma}_{u}} \boldsymbol{N}^{T} \boldsymbol{R}^{n} \boldsymbol{a} \boldsymbol{G} \mathrm{d} s, \quad \boldsymbol{K}_{3}=\int_{\tilde{\Gamma}_{u}} \boldsymbol{N}^{T} \boldsymbol{N} \mathrm{d} s, \\
\boldsymbol{K}_{4}=\int_{\tilde{\Gamma}_{\boldsymbol{u}}}\left(\boldsymbol{N}^{T} \boldsymbol{R}^{d} \boldsymbol{G}-\boldsymbol{N}^{T} \overline{\overline{\boldsymbol{G} \boldsymbol{u}^{* \text { node }}}} \boldsymbol{N}\right) \mathrm{d} s, \quad \boldsymbol{K}_{5}=\int_{\tilde{\Gamma}_{u}} \boldsymbol{G}^{T}\left[\left(\boldsymbol{R}^{d}\right)^{T}-\left(\boldsymbol{r}^{u}\right)^{T}\right] \boldsymbol{N} \mathrm{d} s, \\
\boldsymbol{K}_{6}=\int_{\tilde{\Gamma}_{\boldsymbol{u}}}\left[\boldsymbol{G}^{T}\left(\boldsymbol{R}^{d}\right)^{T} \boldsymbol{R}^{d} \boldsymbol{G}-\boldsymbol{G}^{T}\left(\boldsymbol{R}^{d}\right)^{T} \overline{\overline{\boldsymbol{G} \boldsymbol{u}^{* \text { node }}}} \boldsymbol{N}-\boldsymbol{G}^{T}\left(\boldsymbol{r}^{u d}\right)^{T} \boldsymbol{N}\right] \mathrm{d} s, \quad \boldsymbol{K}_{7}=\int_{\tilde{\Gamma}_{u}} \boldsymbol{G}^{T}\left(\boldsymbol{r}^{u D}\right)^{T} \boldsymbol{N} \mathrm{d} s .
\end{array}\right.
$$

It should be noted that we here adopt a general form of the tangent stiffness matrix $\boldsymbol{K}_{1}$ in (24) instead of splitting it into the so-called material and geometric stiffnesses $[9,12]$.

In the implicit MPM, the governing equations are solved at the nodes of the background grid and the material points work as the integration points in the finite element method (FEM). Similar to the FEM, we need to assemble the global stiffness matrix and the force vector involving the volume integrals over each element and the surface integrals over the bounds of the elements. Generally, the volume integral of a function $f(\boldsymbol{x})$ appearing (23) and (24) over an element $e$ is calculated in the implicit MPM as:

$\int_{\tilde{\mathcal{B}} e} f(\boldsymbol{x}) \mathrm{d} v=\sum_{p=1}^{N_{p}} f\left(\boldsymbol{x}_{p}\right) v_{p}$,

where $\tilde{\mathcal{B}}^{e}$ is the volume of the element $e, N_{P}$ is the number of material points within the element and $v_{p}$ is the volume represented by point $p$. For example, the formulation to compute the $f_{\text {int } 1}$ at node $I$ is:

$f_{\text {int1I }}=\sum_{p=1}^{N_{p}} \nabla^{\boldsymbol{x}} N_{I}\left(\boldsymbol{x}_{p}^{n}\right) \cdot \boldsymbol{\sigma}_{p}^{n} \operatorname{det}\left(F_{\Delta}\right) v_{p}^{n}$,

where $n$ indicates the $n$th step. The surface integrals are calculated by using the regular Gaussian quadrature scheme with $2 \times 2$ integration points for each bound square. We adopt the Newton-Raphson iteration to solve the incremental equilibrium problems. Once we achieve the convergent solution of displacement field, the position of material points is updated as follows:

$\boldsymbol{x}_{p}^{n+1}=\boldsymbol{x}_{p}^{n}+\sum_{I=1}^{N_{e}} \boldsymbol{u}_{I} N_{I p}$,

where $I$ is the $I$ th node of the element containing point $p$, $\boldsymbol{u}_{I}$ is the displacement within the load step, $N_{I p}=N_{I}\left(\boldsymbol{X}_{p}^{n}\right)$ and $N_{e}$ is the number of nodes per element, e.g., $N_{e}=8$ for the linear cube element.

\section{Semi-Lagrangian nonlocal damage model}

We introduce a semi-Lagrangian nonlocal damage model to replicate the behaviors of an isotropic quasi-brittle material in the geometrically nonlinear regime. This model is an extension of the isotropic nonlocal damage model for the infinitesimal range originally proposed by Jirásek [20]. In particular, we introduce a logarithmic-exponential mapping that enables us to build connections between models with infinitesimal kinematics and those with multiplicative kinematics. Then, we introduce a semi-Lagrangian approach to capture the size-independent responses without considering the deformation of nonlocal influence regions in the finite strain range. The major upshot of these two treatments is the simplicity and the ease for implementation. While the logarithmic-exponential mapping allows us to extend existing small-strain models to replicate large deformation responses, the semi-Lagrangian approach greatly simplifies the calculation of the nonlocal strain measure while circumventing the pathological mesh dependence commonly exhibited in the rate-independent local damage model [10].

\subsection{Preprocessing step: establishing connections between infinitesimal and multiplicative kinematics}

Given the information of the end of $n$th loading step, we can obtain the current deformation gradient $\boldsymbol{F}$ by combining incremental deformation gradient $\boldsymbol{F}_{\Delta}$ and $\boldsymbol{F}_{n}$, as illustrated in (14). We then compute the left Cauchy-Green tensor as $\boldsymbol{B}=\boldsymbol{F} \boldsymbol{F}^{T}$. Finally, the logarithmic strain measure that reads

$\boldsymbol{\varepsilon}=\frac{1}{2} \ln \boldsymbol{B}$,

is computed. This logarithmic strain measure is used as the input for a small-strain constitutive law to obtain a kinetic measure as described in Sect. 3.2.

\subsection{Strain update: continuum damage theory}

We consider a strain-based damage model at the tangent of multiplicative kinematics. Here, we employ the effective 
stress theory, which hypothesizes that there exists an imaginary undamaged material that experiences the same strain history of the real counterpart material but exhibits pathindependent behavior characterizes by a strain energy density $W\left(\varepsilon_{I}, \varepsilon_{I I}, \varepsilon_{I I I}\right)$ and the constitutive responses of the two materials are related by a scalar damage parameter, such that

$\boldsymbol{\tau}=(1-\omega) \frac{\partial W}{\partial \boldsymbol{\varepsilon}}$,

where $\tau$ is the Kirchhoff stress and $\omega$ is the damage variable. We assume the elastic responses of both materials are linear such that $\boldsymbol{D}^{e}=\partial^{2} W / \partial \boldsymbol{\varepsilon} \partial \boldsymbol{\varepsilon}$ and $\boldsymbol{D}^{e}$ therefore is the elastic material stiffness tensor.

This damage parameter is then governing by a phenomenological exponential damage law, i.e.,

$\omega=g(\kappa)=1-\frac{\varepsilon_{0}}{\kappa} \exp \left(-\frac{\left\langle\kappa-\varepsilon_{0}\right\rangle}{\varepsilon_{f}-\varepsilon_{0}}\right)$,

and loading-unloading conditions

$f(\varepsilon, \kappa) \equiv \varepsilon_{\mathrm{eq}}(\boldsymbol{\varepsilon})-\kappa \leq 0, \quad \dot{\kappa} \geq 0, \quad f(\boldsymbol{\varepsilon}, \kappa) \dot{\kappa}=0$,

in which $g$ is the damage evolution function, $\kappa$ is an internal variable that corresponds to the maximum level of equivalent strain ever reached in the previous history of the material, $\varepsilon_{0}$ is the limit elastic strain, $\varepsilon_{f}$ is a parameter that controls softening, the brackets $\langle\cdot\rangle$ denote the positive part operator, defined by the relations $\langle a\rangle=a$ for $a>0$ and $\langle a\rangle=0$ for $a \leq 0, f$ is the damage loading function, $\varepsilon_{\mathrm{eq}}$ is a scalar measure of the strain level called the equivalent strain and defined as [31]:

$\varepsilon_{\text {eq }}=\sqrt{\sum_{I=1}^{3}\left\langle\varepsilon_{I}\right\rangle^{2}}$,

where $\left\langle\varepsilon_{I}\right\rangle$ are the positive parts of principal values of the strain tensor $\boldsymbol{\varepsilon}$.

Generally speaking, the nonlocal approach consists in replacing a certain variable by its nonlocal counterpart obtained by weighted averaging over a spatial neighborhood of each point under consideration. The weighted spatial average of the local value $\bar{\varepsilon}_{\text {eq }}$ is defined as

$\bar{\varepsilon}_{\mathrm{eq}}(\boldsymbol{x})=\int_{V} \alpha(\boldsymbol{x}, \boldsymbol{\eta}) \varepsilon_{\mathrm{eq}}(\boldsymbol{\eta}) \mathrm{d} \boldsymbol{\eta}$,

where $\alpha(\boldsymbol{x}, \boldsymbol{\eta})$ is a given nonlocal weight function. Considering of eliminating the influence of boundaries, the weight function is usually rescaled such that the nonlocal operator does not alter a uniform field, by setting $\alpha(\boldsymbol{x}, \boldsymbol{\eta})=\frac{\alpha_{0}(\|\boldsymbol{x}-\boldsymbol{\eta}\|)}{\int_{V} \alpha_{0}(\|\boldsymbol{x}-\boldsymbol{\xi}\|) \mathrm{d} \boldsymbol{\xi}}$,

where $\alpha_{0}(r)$ is a monotonically decreasing nonnegative function of the distance $r=\|\boldsymbol{x}-\boldsymbol{\eta}\|$. The weight function here is taken as the truncated quartic polynomial function

$\alpha_{0}(r)=\left\langle 1-\frac{r^{2}}{R^{2}}\right\rangle^{2}$

where $R$ is a parameter related to the internal length scale. One important departure of this constitutive law is that the weighting function that generates the nonlocal strain invariant measure in Eq. (33) is described in the current configuration and does not deform in each incremental step. As a result, the influence zone in Eq. (35) remains spherical and does not deform according to the deformation map, a feature mimicking the semi-Lagrangian kernel used in Guan et al. [15] and Guan et al. [16].

In the loading-unloading conditions, $\varepsilon_{\mathrm{eq}}$ in (31) is replaced by its weighted spatial average, such that

$\bar{\varepsilon}_{\text {eq }}-\kappa \leq 0, \quad \dot{\kappa} \geq 0, \quad\left(\bar{\varepsilon}_{\text {eq }}-\kappa\right) \dot{\kappa}=0$.

In the implementation, searching neighboring particles for a specific particle could be cumbersome. We here take advantage of the structured background grid and construct multiple sets to improve the efficiency of such searching. The idea of the searching procedure is illustrated in a two-dimensional example shown in Fig. 3.

Let us denote the set of all material points in the domain as $S_{0}$. The neighbor particles of a specific (target) particle comprise a set of material points within a circle domain within radius $R$. Here, instead of looping over the entire set $S_{0}$ to identify the material points within the neighborhood of each target material point, we first create a subset of material points by expanding the initial neighborhood with a positive factor $c$ to create a subset of $S_{0}$ that reads

$S_{\mathrm{pot}}=\left\{\boldsymbol{x}_{p}|| \boldsymbol{x}_{p}-\boldsymbol{x}_{t} \|<c R\right\}$,

where $c>1$ is a constant, $R$ is the internal length scale parameter and $\boldsymbol{x}_{t}$ is the position vector of a point in the spatial (current) coordinates. Then, the Euclidean distances between the target material point (red dot in Fig. 3) and all material point in the set $S_{\text {pot }}$ are computed and those material points with distance less than $R$ are then sorted into the neighborhood set of the target particle. We only construct $S_{\text {pot }}$ one time by setting $c$ as a large parameter consisting of the deformation of the body. Since $S_{\text {pot }}$ contains only a portion of material points in $S_{0}$, this approach can significantly reduce the total number of arithmetic operations required to construct the nonlocal measure. 
Fig. 3 Hierarchy sets for searching neighboring particles of a specific particle. $S_{0}, S_{\text {cell }}$ and $S_{\text {pot }}$ are one-time construction. $n=[c R / h]$ is an integer determined by the ratio of $c R$ to the mesh size $h$. (Color figure online)

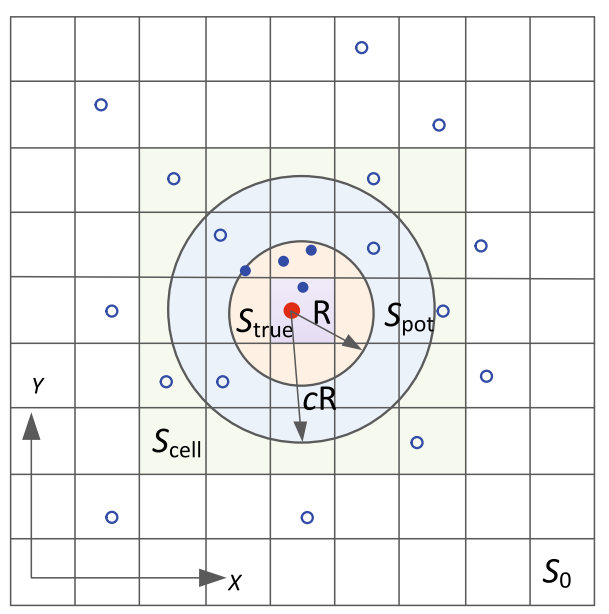

- Target particle $\mathbf{x}_{t}$

O Material points having zero weight

- Material points having non-zero weights

Cell containing the target particle $(I, J)$

Cells containing the potential neighborhood particles (I-n: I+n,J-n: J+n) $\Omega_{\text {cell }}$

$S_{0}=\left\{\mathbf{x}_{p} \mid \mathbf{x}_{p} \in B\right\}$

$S_{\text {cell }}=\left\{\mathbf{x}_{p} \mid \mathbf{x}_{p} \in \Omega_{\text {cell }}\right\} \subset S_{0}$

$S_{\text {pot }}=\left\{\mathbf{x}_{p} \mid\left\|\mathbf{x}_{p}-\mathbf{x}_{t}\right\|<c R c>1\right\} \subset S_{\text {cell }}$

$S_{\text {true }}=\left\{\mathbf{x}_{p} \mid\left\|\mathbf{x}_{p}-\mathbf{x}_{t}\right\|<R\right\} \subset S_{\text {spot }}$
Nevertheless, a naïve approach that directly obtains $S_{\text {pot }}$ could still be time-consuming. Hence, we utilize the structured background meshes to simplify the search. Mincing a simple technique commonly used in finite difference method, we assign each element with an identity as $\Omega_{(i, j)}$, where $\Omega$ denotes the region of the element, and $i$ and $j$ are the indices along $x$ - and $y$-axes, respectively. For the target particle belonging to a cell $\Omega_{(I, J)}$, the set $S_{\text {pot }}$ is a subset of $S_{\text {cell }}=$ $\left\{x_{p} \mid x_{p} \in \bigcup \Omega_{i, j}, i \in[I-n: I+n], j \in[J-n, J+n]\right\}$, i.e., $S_{\text {pot }} \subset S_{\text {cell }}$, where $n=[c R / h]$ is an integer determined by the ratio of $c R$ to the mesh size $h$. The reason why we introduce $S_{\text {cell }}$ is that the particles $\boldsymbol{x}_{p} \in S_{\text {cell }}$ are easily determined since the background cells are orderly arranged, and therefore, the search domain for $S_{\text {pot }}$ would dramatically reduce. During the computation, we only need to loop the particles belonging to $S_{\text {pot }}$ to determine the particles with nonzero weights. We thus significantly speed up the construction of influence zone or neighborhood for the nonlocal damage model.

\subsection{Postprocessing step: updates stress and semi-implicit tangential stiffness}

Once we obtain the Kirchhoff stress $\tau$ via (29), the Cauchy stress $\sigma$ can be obtained via

$\sigma=J^{-1} \tau$

We now give the specific formulation of spatial tangential tensor $\boldsymbol{a}$ in (35) by applying the chain rule [9,11,34],

$a_{i j k l}=\frac{1}{2 J}[\boldsymbol{D}: \boldsymbol{L}: \boldsymbol{H}]_{i j k l}-\sigma_{i l} \delta_{j k}$,

where

$\boldsymbol{D}=\frac{\partial \boldsymbol{\tau}}{\partial \boldsymbol{\varepsilon}}=(1-\omega) \boldsymbol{D}^{e}, \quad \boldsymbol{L}=\frac{\partial \ln \boldsymbol{B}}{\partial \boldsymbol{B}}$.
It should be mentioned that a consistent tangent $\boldsymbol{D}$ should include the nonlocal contribution. However, this nonlocal contribution is omitted in our calculation to simplify the implementation. Consequently, the convergent rate in the Newton-Raphson iterations is negatively influenced by such an inconsistent tangent. The derivative term $\partial \ln \boldsymbol{B} / \partial \boldsymbol{B}$ can be obtained via Taylor series expansion or spectral decomposition [37]. In the invariant notation, $\boldsymbol{L}=\partial \ln \boldsymbol{B} / \partial \boldsymbol{B}$ reads

$\boldsymbol{L}=\sum_{a=1}^{n s d} \sum_{b=1}^{n s d} g\left(\lambda_{a}, \lambda_{b}\right) \boldsymbol{u}_{a} \otimes \boldsymbol{u}_{b} \times \boldsymbol{u}_{a} \otimes \boldsymbol{u}_{b}$

where

$g\left(\lambda_{a}, \lambda_{b}\right)=\frac{2\left(\ln \lambda_{b}-\ln \lambda_{a}\right)}{\lambda_{b}^{2}-\lambda_{a}^{2}}$ if $\lambda_{b} \neq \lambda_{a}$

and

$g\left(\lambda_{a}, \lambda_{b}\right)=\frac{1}{\lambda_{a}^{2}}$ otherwise,

where $\lambda_{a}$ is the principal stretch and $\boldsymbol{u}_{a}$ is the corresponding direction (i.e., the normalized eigenvector of the symmetric tensor $\boldsymbol{B}$ ). The components form of $\boldsymbol{H}$ in Eq. (39) reads

$H_{i j k l}=\delta_{i k}(\boldsymbol{B})_{j l}+\delta_{j k}(\boldsymbol{B})_{i l}$.

In summary, we adopt an isotropic nonlocal damage model with integral generalization to model the finite deformation of quasi-brittle materials. The model is verified via an example of a bar subjected to a uniaxial loading. The details can be found in "Appendix". 


\section{Internal variable recovery}

Since all internal variables and stress measures are stored in the material points, we modify the method originally proposed in [44] where moving least squares (MLS) is used to project the data stored in a point cloud onto a smooth field. This treatment is required for stress analysis [32,60], adaptive simulations [59] and visualization of data.

In the MLS, the reconstruction function of a scalar field $u^{h}(x)$ in one dimension is assumed as holding the following form:

$u^{h}(x, \bar{x})=a_{0}(\bar{x})+a_{1}(\bar{x}) x+\cdots+a_{r}(\bar{x}) x^{r}=\boldsymbol{P}^{T}(x) \boldsymbol{a}(\bar{x})$,

where the coefficients of the monomials $\boldsymbol{P}^{T}(x)=\left[1, x, x^{2}\right.$, $\left.\ldots, x^{r}\right]$, depend on the spatial coordinate $\bar{x}$, i.e., $\boldsymbol{a}=\boldsymbol{a}(\bar{x})$, $r$ is the maximum degree of the monomials and a bar over $x$ for $\boldsymbol{a}$ is adopted to distinguish $x$ for $\boldsymbol{P}$. The general idea of the MLS is to find $\boldsymbol{a}(\bar{x})$ so that the approximation function $u^{h}(x, \bar{x})$ minimizes the following functional:

$I(\boldsymbol{a})=\sum_{p=1}^{n p} w\left(\bar{x}-x_{p}\right)\left[u_{p}^{h}\left(x_{p}, \bar{x}\right)-u_{p}\right]^{2}$,

where $p$ represents the $p$ th particle in $n p$ particles, $w\left(\bar{x}-x_{p}\right)$ is the weighting function of particle $p$ and $u_{p}=u\left(x_{p}\right)$ is the known value at particle $p$. By replacing the $\bar{x}$ in (46) with $x$ and substituting (45) into (46), we obtain that:

$I(\boldsymbol{a})=\sum_{p=1}^{n p} w\left(x-x_{p}\right)\left[\boldsymbol{P}^{T}\left(x_{p}\right) \boldsymbol{a}(x)-u_{p}\right]^{2}$.

Take the derivative of $I$ with respect to each component of $\boldsymbol{a}$ and set them to zeros to get:

$\boldsymbol{M}(x) \boldsymbol{a}(x)=\boldsymbol{b}(x)$,

where $\boldsymbol{M}(x)$ and $\boldsymbol{b}(x)$ are defined as follows:

$$
\begin{aligned}
\boldsymbol{M}(x) & =\sum_{p=1}^{n p} w\left(x-x_{p}\right) \boldsymbol{P}\left(x_{p}\right) \boldsymbol{P}^{T}\left(x_{p}\right), \\
\boldsymbol{b}(x) & =\sum_{p=1}^{n p} w\left(x-x_{p}\right) \boldsymbol{P}\left(x_{p}\right) u_{p} .
\end{aligned}
$$

The approximation of $u(x)$ thus can be:

$$
u^{h}(x)=\boldsymbol{P}^{T}(x) \boldsymbol{M}^{-1}(x) \boldsymbol{b}(x) .
$$

For three-dimensional problems, we employ linear polynomials as the basis for the reconstruction, i.e., $\boldsymbol{P}^{T}(\boldsymbol{x})=$ $\left[1, x_{1}, x_{2}, x_{3}\right]$. We choose the Euclidean distance between the material point $\boldsymbol{x}_{p}$ and the desired interpolation point $\boldsymbol{x}$ as the input of the weighting functions, as opposed to the taxicab distance originally proposed in Gong [14]. This treatment is necessary to ensure that the resultant projected field from the point cloud is invariant against rotation and the choice of reference coordinate system [57,58]. We further adopt a cubic spline as the weighting function:

$w\left(\boldsymbol{x}-\boldsymbol{x}_{p}\right)=\left\{\begin{array}{lr}\frac{2}{3}-4 r^{2}+4 r^{3} & r \leq \frac{1}{2} \\ \frac{4}{3}-4 r+4 r^{2}-\frac{4}{3} r^{3} & \frac{1}{2} \leq r \leq 1 \\ 0 & r \geq 1,\end{array}\right.$

where $r=\left\|\boldsymbol{x}-\boldsymbol{x}_{\boldsymbol{p}}\right\| /(2 h)$ and $h$ is the grid spacing and $\|\cdot\|$ denotes the Euclidean norm.

\section{Numerical examples}

We present three numerical examples to verify the SBMPM and to demonstrate the ability of the proposed method to handle path-dependent behaviors of objects of complex geometries. In all examples, the full Newton-Raphson algorithm is selected to solve the incremental equilibrium problem implicitly.

\subsection{Sphere under isotropic compression and rigid body rotation}

As shown in Fig. 4, the true domain is a sphere with a radius of $2.2 \mathrm{~m}$ and a center at $(5,5,5) \mathrm{m}$. The size of the background mesh is $1 \mathrm{~m}$, which means the surrogate domain can be a cube with the size of $2 \times 2 \times 2 \mathrm{~m}$. We consider three cases: (1) rigid body rotation around the center axis along the $z$-axis; (2) isotropic compression; and (3) isotropic compression with rigid body rotation around the center axis along the $z$-axis. The surrogate domain thus can be kept constant

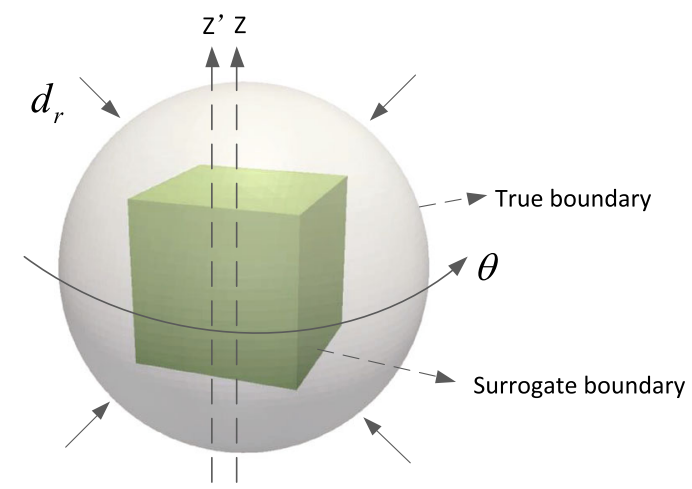

Fig. 4 Surrogate domain of a sphere 


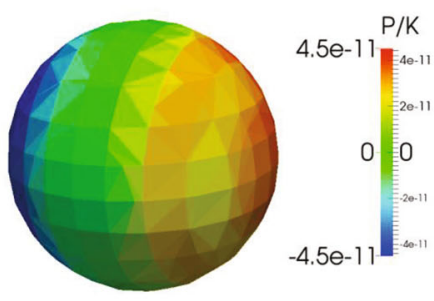

$\theta=5^{\circ}$

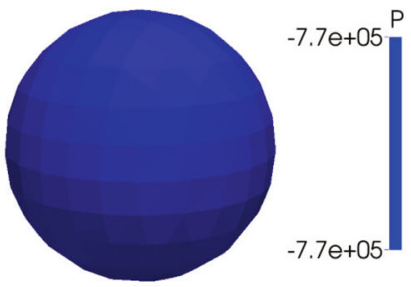

$d_{r}=0.01$

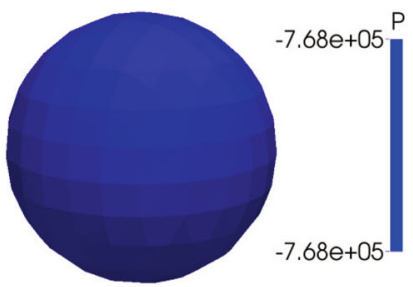

$\theta=5^{\circ} d_{r}=0.01$

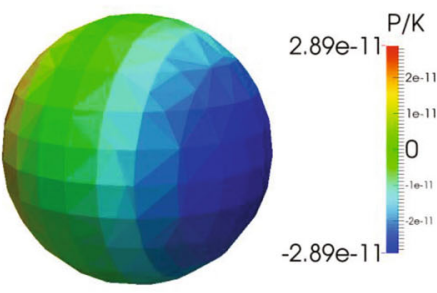

$\theta=10^{\circ}$

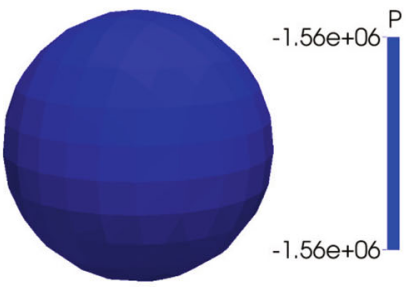

$d_{r}=0.02$

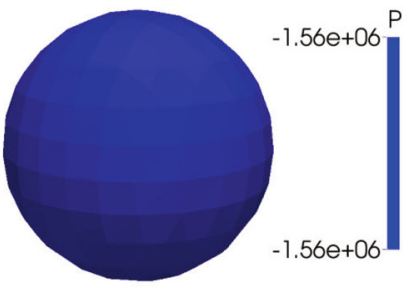

$d_{r}=0.02$

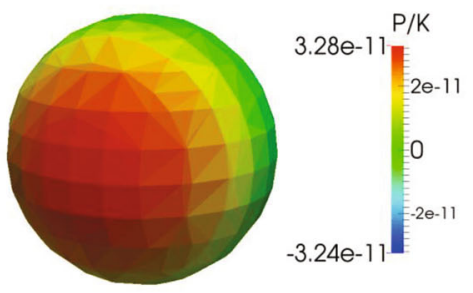

$\theta=15^{\circ}$

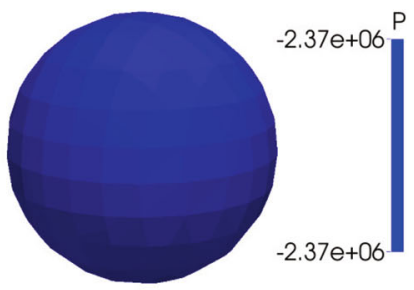

$d_{r}=0.03$

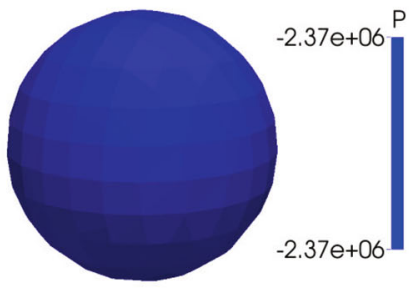

$d_{r}=0.03$

Fig. 5 Distributions of pressure for different boundary conditions [P: hydrostatic pressure (Pa) and K: bulk modulus (Pa)]

since the sphere does not change its shape for rigid body rotation and isotropic compression. The incremental rotation angle and radial displacement in each loading step are $5^{\circ}$ and $0.01 \mathrm{~m}$, respectively. We investigate the first three loading steps. We initially set 27 material points within each element, and therefore, 216 material points in total are adopted to represent the surrogate domain. The material is assumed as isotropic hyperelastic and described by the Hencky model. The Young's modulus is $100 \mathrm{MPa}$, and the Poisson's ratio is 0.2 . The convergence criterion is that the residual is less than $1 \times 10^{-12} \mathrm{~N}$ or the relative displacement change to the displacement obtained in the first iteration is less than $1 \times 10^{-9}$.

Figure 5 shows the distributions of pressure for different boundary conditions. For pure rigid body rotation (the first row), we can see that the maximum dimensionless pressure is only $4 \times 10^{-11}$, which indicates that the material points are stress-free for rigid body rotation. For pure isotropic compression (the second row), the distributions of the pressure are homogeneous as expected. When we combine the rigid body rotation and the isotropic compression, the stress is almost the same as the isotropic compression. (The maximum relative error is approximate $0.26 \%$.)
As shown in Fig. 4, the $z$-axis is a vertical axis passing through the center point of the sphere, e.g., $(5,5,5)$. We now move the rotation axis form $z$ to $z^{\prime}$, which is a vertical axis passing through the point $(4.8,4.8,5)$. The rigid body rotation thus is unsymmetrical within the body. As a comparison to the SBM, we do not implement the SBM and directly impose the Dirichlet boundary conditions from the true boundary to its closest nodes of the background grid. Note that since the unsymmetrical rotation would evolve the true boundary, we here only consider one loading step; otherwise, we need to update the surrogate domain. Figure 6 compares the dimensionless pressures with the SBM and without the SBM. We can see that the material points are almost stress-free with the SBM under such an unsymmetrical rigid body rotation. However, without the SBM, the maximum dimensionless pressure is approximate 0.005 after unsymmetrical rigid body rotation of $5^{\circ}$. The advantage of the proposed method over the traditional treatment of imposing of the Dirichlet boundary conditions in the MPM is demonstrated through this comparison. 
Fig. 6 Comparison of pressures after an unsymmetrical rigid rotation of $5^{\circ}:$ a with the SBM and $\mathbf{b}$ without the SBM

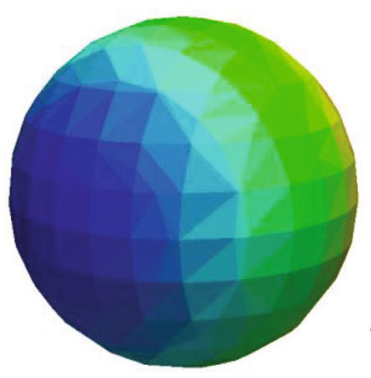

(a)
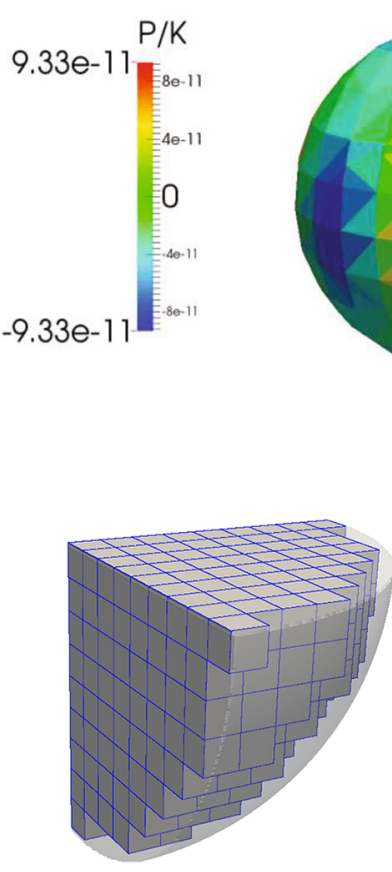

(a)
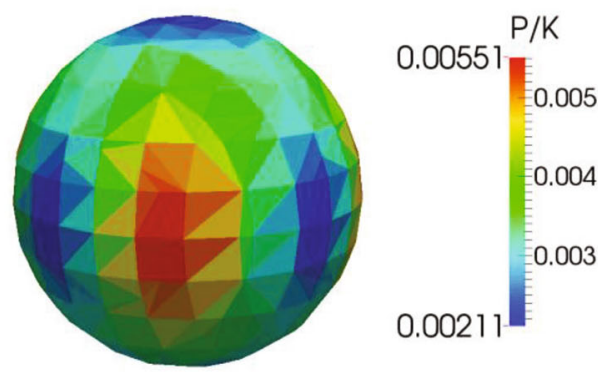

(b)

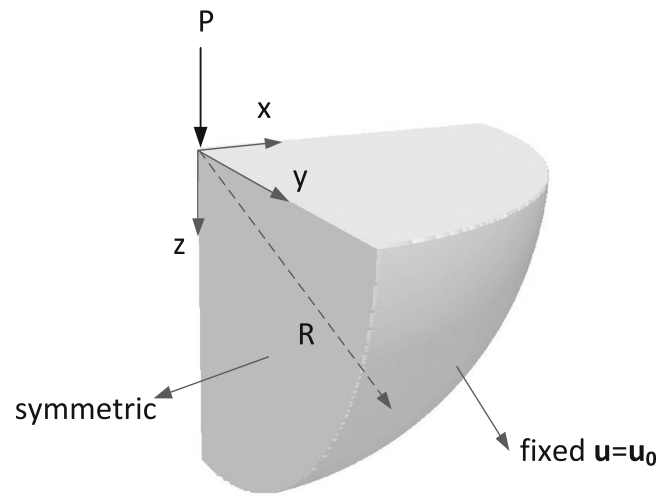

Fig. 7 Numerical model for Boussinesq's problem

\subsection{Boussinesq's problem}

In this example, we adopt the Boussinesq's problem to assess the accuracy of the proposed method by comparing the numerical solutions with the analytical/exact solution of Boussinesq's problem. In the Boussinesq's problem, there is a point load acting normal to the surface of an elastic halfspace. We can achieve the analytical solution of the field of displacement in cylindrical coordinates. As shown in Fig. 7, we here consider the symmetries of the problem and only concern a quarter half-sphere with a radius of $R$. The point load with magnitude $P=1000 \mathrm{~N}$ is applied at the origin of coordinates on the quarter half-sphere. The $z$-axis represents depth beneath the ground surface. We set symmetric boundary conditions for the planes of $x=0$ and $y=0$. The displacement on the sphere surface is set as the analytical solution and be shifted to apply on surrogate domains. If the shifted scheme works, the displacement along z-axis needs to be consistent with the analytical solution. The material parameters are Young's modulus $100 \mathrm{MPa}$ and Poisson's ratio 0.2 .

We first estimate the influence of the surrogate domain on accuracy. As shown in Fig. 8, we employ two surrogate domains with different mesh sizes of $h=1$ and $0.5 \mathrm{~m}$, respectively, for a fixed true domain with $R=9.2 \mathrm{~m}$. The vertical displacement along $z$-axis for these two domains is com-
Fig. 8 Two surrogate domains for a quarter half-sphere with a mesh size $1 \mathrm{~m}$ and $\mathbf{b}$ mesh size $0.5 \mathrm{~m}$

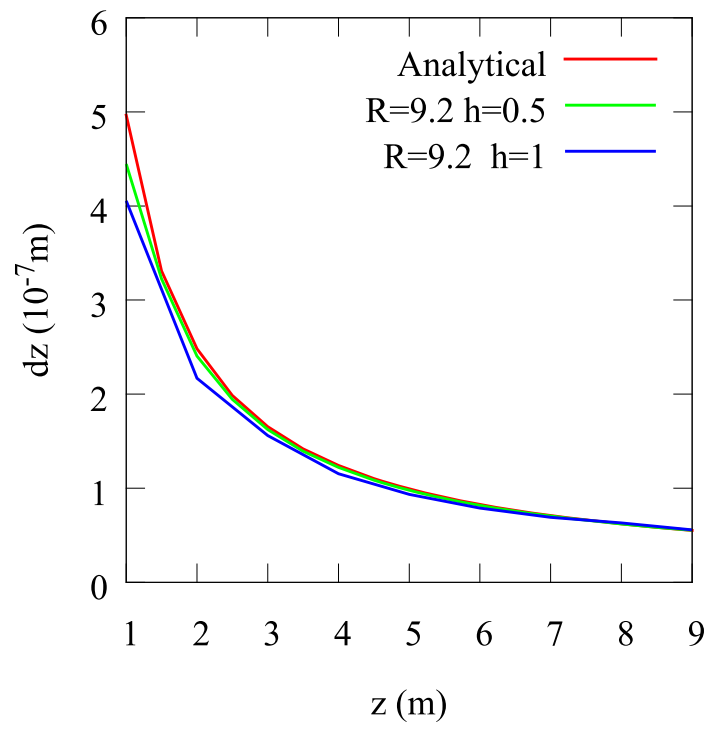

Fig. 9 Comparisons of analytical results and numerical results: different surrogate domains for a identical true domain

pared with the analytical solution, as shown in Fig. 9. Note that the analytical solution for the origin point is infinite and we therefore only compare the region of $z \in[1: 9]$. We can see that numerical solutions are pretty close to the analytical solution even for the coarse domain with $h=1 \mathrm{~m}$.

We then fix the coarse surrogate domain to represent various true domains with different sizes. Note that the shapes 


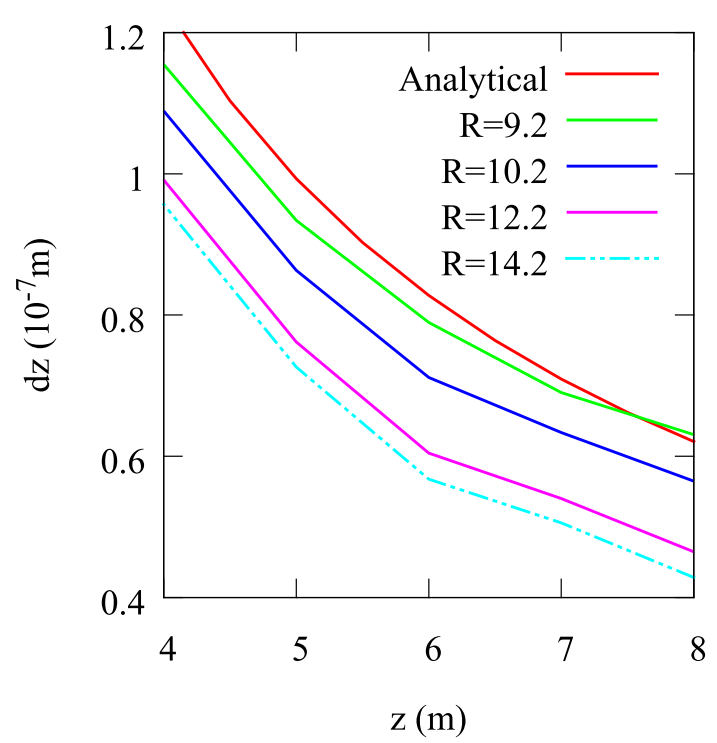

Fig. 10 Comparisons of analytical results and numerical results: different true domains with an identical surrogate domain

of these true domains are identical, i.e., a quarter of a halfsphere. As illustrated in Fig. 10, we can see that the error of the displacement along $z$-axis increases with the radius of the true domain. This is caused by the fact that the essence of the SBM is to approximate the displacement on the true boundary via its first-order Taylor expansion at the surrogate boundary. The accuracy of this approximation decreases with the distance between the true boundary and the surrogate boundary.

In summary, without using a conformal grid, we can effectively shift the boundary conditions applied on the true boundary to a boundary of an inside surrogate domain composing of structured cells.

\subsection{Sand grain subjected to distributed loads}

In this example, we implement the proposed method to study the mechanical behavior of a quasi-brittle sand grain subjected to Dirichlet boundary conditions. As shown in Fig. $11 \mathrm{a}$, we represent the true boundary of the grain by a set of triangles based on a binarized 3D image of Hostun sand [19]. This image is provided to us by Drs. Edward Ando and Max Miebicke from the laboratory 3SR in Grenoble, France. As a part of larger ongoing efforts to promote third-party verification, validation and falsification of numerical models as documented in Gupta et al. [19], the original micro-CT images, the binarized image which we used in this study and the conformal tetrahedral volume mesh and the conformal triangle surface mesh are all available from the repository in Mendeley data (cf. Gupta et al. [18]).

The characteristic spatial length is enlarged by 100 times than that of the original image and holds the value of $100 \mathrm{~mm}$. We then initialize the signed distance field at a structured

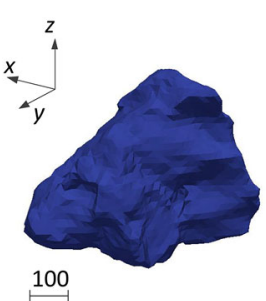

(a)

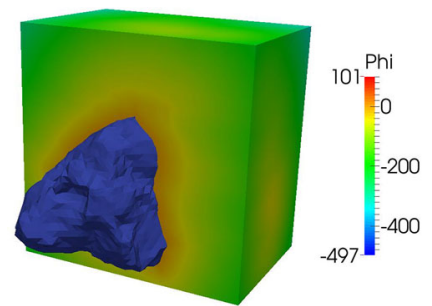

(b)
Fig. 11 Grain model. a Grain image (unit: $\mathrm{mm}$ ) and $\mathbf{b}$ signed distance field

background mesh. A half-space of the signed distance field is illustrated in Fig. 11b. Note that the true boundary is with $\phi=0$.

Once we obtain the field of the signed distance function, we can generate a surrogate domain composing of structured elements by the algorithm previously discussed. To assess the sensitivity of the numerical solution with respect to the choices of the surrogate domains, we solve the boundary value problems three times, each with a different surrogate domain of a slightly different mesh size, i.e., $h=20,22$ and $25 \mathrm{~mm}$. Figure 12 shows these surrogate domains. It shows that a moderate variation in the mesh size may lead to a change in the generated surrogate domain. This is attributed to the fact that the surrogate domains composing of voxels are constrained to be inside the true domain. Note that the total number of degrees of freedom for these three surrogate domains is $10,263,7653$ and 5163 , respectively. It is obvious that generating such a simple grid is much more efficient than generating a conformal grid required in the traditional FEM. In the proposed method, the most significant procedure is to set up the map between the surrogate boundary and the true boundary. The insets of Fig. 12 also show the enlargements at the top regions of maps between the surface Gauss quadrature points (in red) on the surrogate boundary and their closest points (in blue) on the true boundary.

The boundary conditions for the true boundary are applied as follows. In terms of the geometry of the grain, we specify two reference points $\left(x_{t}, y_{t}, z_{t}\right)=(300,300,450) \mathrm{mm}$ at the top region and $\left(x_{b}, y_{b}, z_{b}\right)=(300,400,80) \mathrm{mm}$ at the bottom region. The spatial vector connecting these two points thus is $t=(0,-0.261,0.965)$. Since the Dirichlet boundary conditions are applied on the true boundary, we denote to the spatial position of a mapped point $p$ on the true boundary as $\boldsymbol{x}_{p}=\left(x_{p}, y_{p}, z_{p}\right)$. If $z_{p}>z_{t}\left(\right.$ or $\left.z_{p}<z_{b}\right)$, we compute the length $R_{p}=\sqrt{\left(x_{p}-x_{t}\right)^{2}+\left(y_{p}-y_{t}\right)^{2}}$ (or $R_{p}=\sqrt{\left(x_{p}-x_{b}\right)^{2}+\left(y_{p}-y_{b}\right)^{2}}$ ). The displacement of the point $p$ is $\boldsymbol{u}_{P}=u_{0}\left\langle R_{0}-R_{P}\right\rangle / R_{0} \boldsymbol{t}$ (or $\boldsymbol{u}_{P}=$ $\left.-u_{0}\left\langle R_{0}-R_{P}\right\rangle / R_{0} t\right)$, where the brackets $\langle\cdot\rangle$ denote the positive part operator, $R_{0}$ is a specified length and $u_{0}$ is the magnitude of displacement. We here set $R_{0}=40 \mathrm{~mm}$ and 


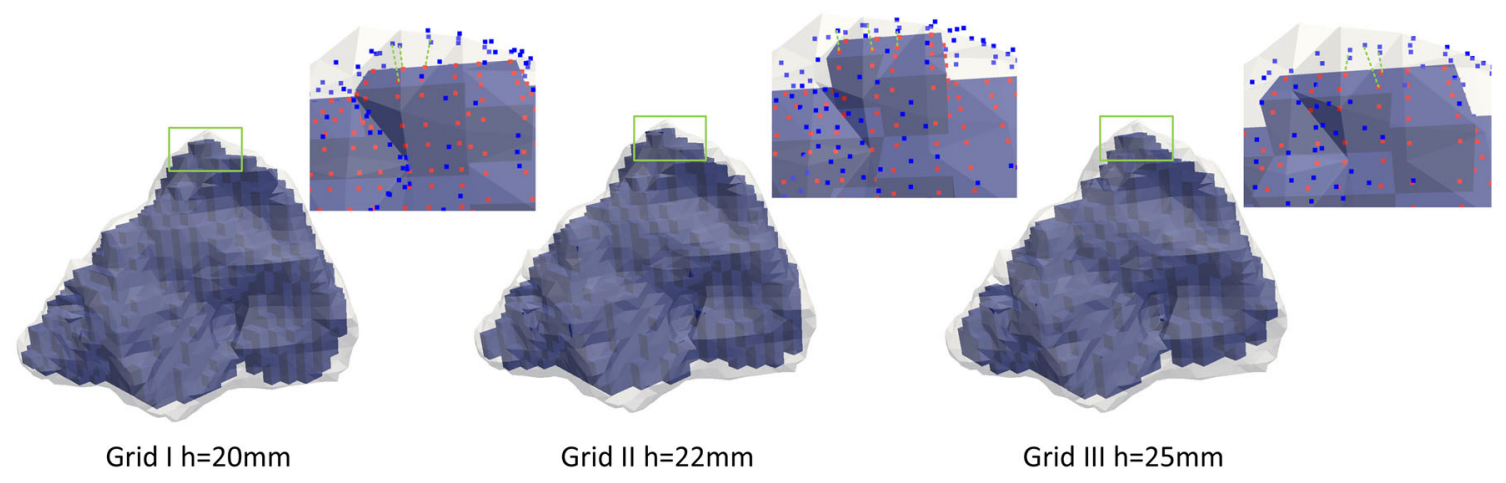

Fig. 12 Surrogate domains with different mesh sizes $h=20,22,25 \mathrm{~mm}$. Insets: maps between the integration points on the surrogate boundary (in red) and the corresponding points on the true boundary (in blue) at the top region. (Color figure online)

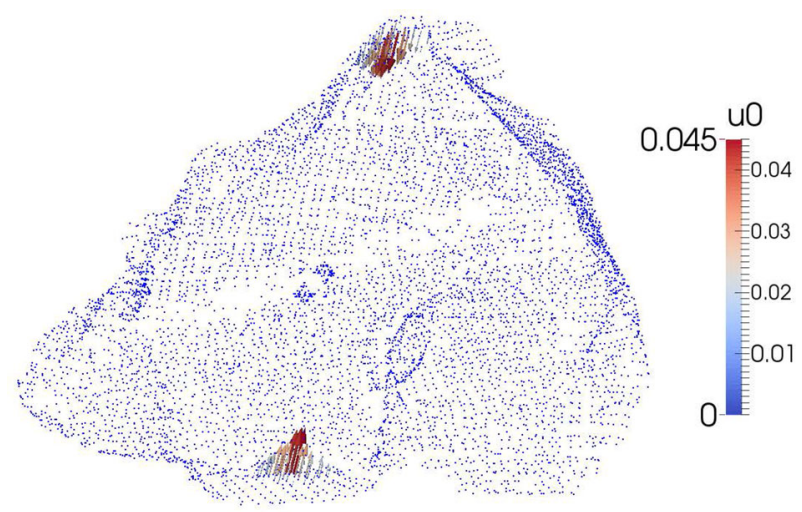

Fig. 13 Locations imposed of boundary conditions (contour denotes the magnitude of displacement vectors)

$u_{0}=0.05 \mathrm{~mm}$. We thus can eliminate the body rotation and the stress concentration at tip of the region subjected to the boundary conditions. The prescribed displacement is shown in Fig. 13.

The constitutive model of the grain is assumed as the nonlocal damage model discussed in Sect. 2.3. The material parameters are given as follows. The elastic modulus is 1 $\mathrm{GPa}$, and the Poisson's ratio is 0.2 . The material parameters in (30) are $\varepsilon_{0}=1 \times 10^{-4}$ and $\varepsilon_{f}=2 \times 10^{-3}$, respectively. The internal length is $R=50 \mathrm{~mm}$. We incrementally increase the loading and adopt the Newton-Raphson iteration to solve the incremental equilibrium problems. Figure 14 shows the evolutions of vertical stress among the horizontal plane of $z=450 \mathrm{~mm}$. We can see that the tendencies of the curves are the same. For each grid, the normal decreases after $u_{\max }=0.2 \mathrm{~mm}$ due to damage evolution of the grain. The discrepancies between the curves for different grids mainly come from the difference of the resolution of the grain and the distance between the true boundary and the surrogate boundary.

Hereafter, we only show the results for the grids with mesh sizes of $h=20$ and $25 \mathrm{~mm}$ since the result for $h=22$ is

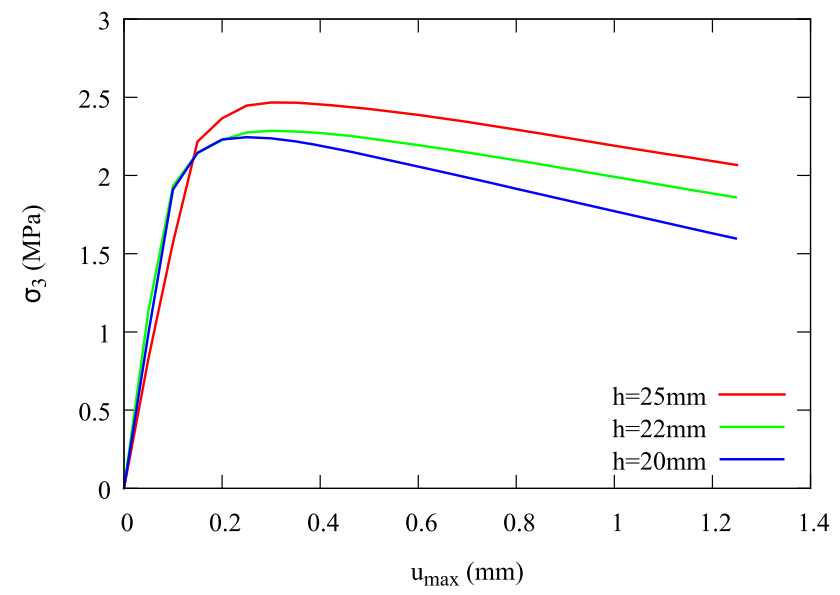

Fig. 14 Evolutions of vertical stress among a horizontal plane with $z=450 \mathrm{~mm}\left(u_{\max }=n u_{0}\right.$ with $n$ denoting $n$th step, and $\sigma_{3}$ is the vertical stress)

almost the same. We first compare the distributions of the von Mises stress for different surrogate domains at the end of the fifth loading step. Figure 15 shows such a comparison with a cutting plane with the normal direction of $\boldsymbol{n}=(1,0,0)$. We can see that the stress concentrates at the region subjected to the Dirichlet boundary conditions. The stress patterns for different surrogate domains are almost the same.

Figure 16 shows the evolutions of the damage parameter $\omega$ under the increasing loading in the plane perpendicular to the $x$-axis. We can see that the damage evolves with the increasing loading. The damage parameters in the regions subjected to the boundary conditions are much higher than in other regions. From the analysis for Fig. 14, for both of the grids, the grain achieves softening states when $u_{\max }=0.25$ $\mathrm{mm}$. We can observe obvious damages when $u_{\max }=0.25$ $\mathrm{mm}$ for each of grids, consistent with the loading curve. Since the grain is three-dimensional, the pattern of damage should be different for various planes. We select four specific planes with different normal directions as $\boldsymbol{n}=$ $(1,0,0),(1,1,0),(0,1,0)$ and $(1,-1,0)$. Figure 17 shows 
Fig. 15 Comparison of von Mises stress $(\mathrm{Pa})$ for two grids at the end of the fifth loading step

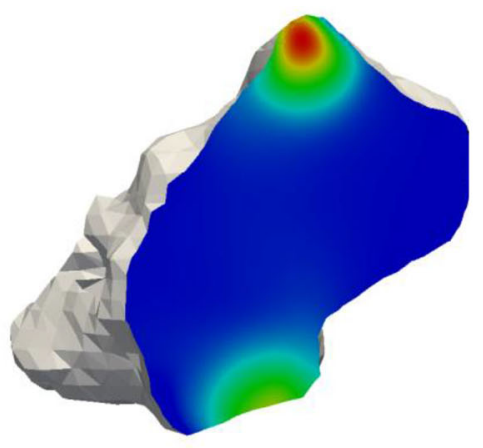

$\mathrm{h}=25 \mathrm{~mm}$

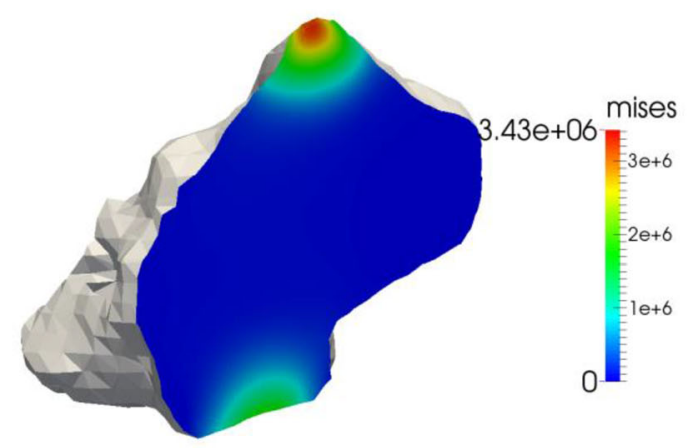

$\mathrm{h}=20 \mathrm{~mm}$
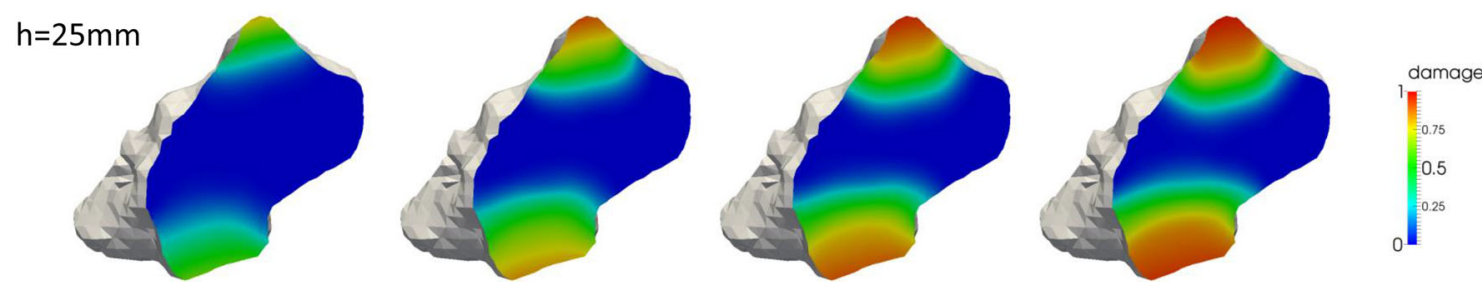

\section{$\mathrm{h}=20 \mathrm{~mm}$}
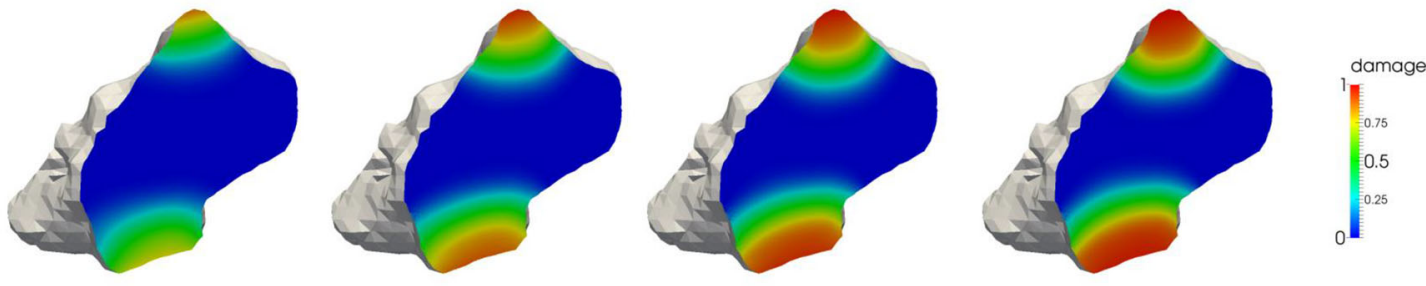

$\mathrm{u}_{\max }=0.25 \mathrm{~mm}$

$$
u_{\max }=0.5 \mathrm{~mm}
$$

$\mathrm{u}_{\max }=0.75 \mathrm{~mm}$

$$
\mathrm{u}_{\max }=1 \mathrm{~mm}
$$

Fig. 16 Evolution of damage parameter for different surrogate domains

\section{$\mathrm{h}=25 \mathrm{~mm}$}
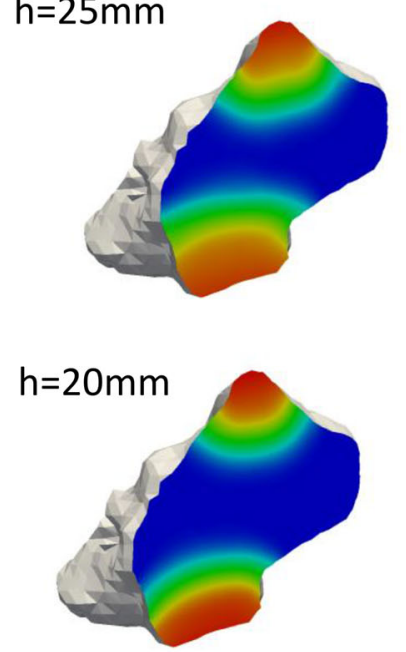

$$
\mathbf{n}=(1,0,0)
$$
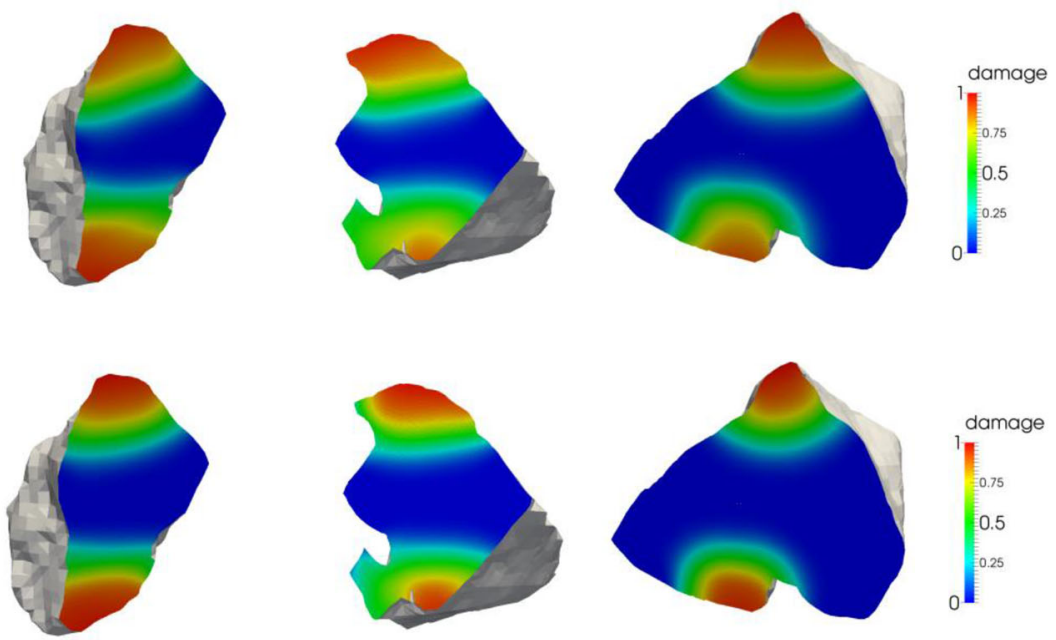

$\mathbf{n}=(1,1,0)$

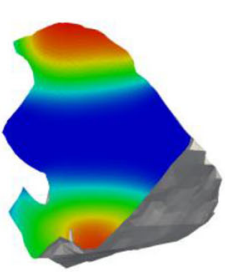

$\mathbf{n}=(0,1,0)$

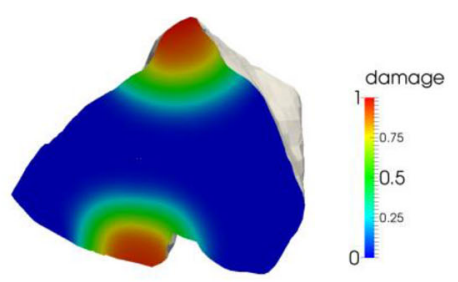

$\mathbf{n}=(1,-1,0)$

Fig. 17 Spatial distributions of damage parameter in different cutting planes ( $\boldsymbol{n}$ is the outward pointing normal of the plane) 
the spatial distributions of damage parameter at these cutting planes when $u_{\max }=1 \mathrm{~mm}$. We can see that the damage patterns are almost the same for different grids.

Given the complexity of replicating damage and fracture at the grain scale and the lack of required data for validation, the objective of this simulation is merely on verifying the implementation of the SBMPM and to ensure that the SBMPM is capable of shifting the boundary conditions from the true boundary to a surrogate boundary. Simulations and validations of different fracture patterns are beyond the scope of this paper. The results of this example indicate that it is feasible to incorporate SBMPM to simplify the image-to-simulation workflow such that simulations can be directly conducted in the voxelized images from 3D MicroCT scanning. The proposed model also provides an alternative approach to simulate the contact between true grains since the complex geometry can be much simplified by the SBMPM.

\section{Conclusion}

In this work, we introduce the shifted boundary material point method designed to model 3D objects of complex geometries undergoing finite deformation. By incorporating the shifted boundary method, the material point integration is conducted in the surrogate domain such that the constraints imposed on the surface of arbitrary geometries can be captured without introducing additional degrees of freedom. To capture the nonlocal constitutive responses and circumvent the loss of material stability upon softening, we extend an isotropic strain-based damage model by incorporating a nonlocal strain measure computed from the long-range interactions among material points within a prescribed range. Numerical examples are used to verify the formulation and to demonstrate a workflow that employs the shifted boundary material point method to replicate deformation of a $3 \mathrm{D}$ particle available in the literature.

In this present work, we constrain our implementations for quasi-static states and the level set is kept constant in the reference configuration. In order to impose traction on the surrogate boundary, the surrogate normal should be decomposed along normal and tangent directions of the true boundary, and the second derivative of the solution is also required. Moreover, future work may also include a formulation that handles the roller boundary conditions to free the tangent displacement. These issues will be addressed in the future. We will also evolve of the level set by solving the Hamilton-Jacobi equation and update the surrogate domain.

Acknowledgements This paper is dedicated to Professor Jiun-Shyan Chen on the occasion of his 60th birthday. This work is supported by the Earth Materials and Processes program from the US Army Research Office under grant contract W911NF-18-2-0306, the Dynamic Materials and Interactions Program from the Air Force Office of Sci- entific Research under grant contract FA9550-17-1-0169, the nuclear energy university program from Department of Energy under grant contract DE-NE0008534 and the Mechanics of Material program at National Science Foundation under grant contract CMMI-1462760. These supports are gratefully acknowledged. The views and conclusions contained in this document are those of the authors and should not be interpreted as representing the official policies, either expressed or implied, of the sponsors, including the Army Research Laboratory or the US Government. The US Government is authorized to reproduce and distribute reprints for government purposes notwithstanding any copyright notation herein.

\section{Appendix}

\section{Linearization}

We linearize all the terms varying with $\boldsymbol{u}$ in (12) to implement the Newton-Raphson iteration. We here only show the procedure of linearization of the terms containing $\sigma=\sigma(\boldsymbol{u})$. There are two main approaches to linearize the weak form of the balance of linear momentum. One is to derive the rate of internal energy using an objective stress rate, in which the tangent stiffness matrix is composed by material tangent stiffness and geometric stiffness, as shown in [4]. In [12], the measurement of stress, such as $\boldsymbol{P}$, is a function of the displacement through the deformation gradient:

$\boldsymbol{P}=\boldsymbol{P}(\boldsymbol{F}(\boldsymbol{u}))$.

The directional derivative of $\boldsymbol{P}$ at $\boldsymbol{u}^{*}$ therefore is:

$D \boldsymbol{P}_{\boldsymbol{u}=\boldsymbol{u}^{*}} \cdot \delta \boldsymbol{u}=\left.\frac{\mathrm{d}}{\mathrm{d} \epsilon}\right|_{\epsilon=0} \frac{\partial \boldsymbol{P}}{\partial \boldsymbol{F}} \frac{\partial \boldsymbol{F}\left(\boldsymbol{u}^{*}+\epsilon \delta \boldsymbol{u}\right)}{\partial \epsilon}=\boldsymbol{A}: \nabla^{\boldsymbol{X}} \delta \boldsymbol{u}$.

To some extent, the tangent modulus $\boldsymbol{A}=\frac{\partial \boldsymbol{P}}{\partial \boldsymbol{F}}$ is in a general form. We can also obtain

$$
\begin{gathered}
D\left[\int_{\mathcal{B}_{0}} \boldsymbol{P}: \nabla^{\boldsymbol{X}} \eta \mathrm{d} V\right] \cdot \delta \boldsymbol{u}=\int_{\mathcal{B}_{0}} \boldsymbol{A}: \nabla^{X} \delta \boldsymbol{u}: \nabla^{\boldsymbol{X}} \eta \mathrm{d} V \\
D\left[\int_{\Gamma_{0}} \boldsymbol{\eta} \cdot \boldsymbol{P} \cdot \boldsymbol{N} \mathrm{d} A\right] \cdot \delta \boldsymbol{u}=\int_{\Gamma_{0}} \boldsymbol{\eta} \cdot \boldsymbol{A}: \nabla^{\boldsymbol{X}} \delta \boldsymbol{u} \cdot \boldsymbol{N} \mathrm{d} A
\end{gathered}
$$

Since the material and spatial virtual work functional are equivalent, we can transform (55) and (56) into

$$
\begin{aligned}
D\left[\int_{\mathcal{B}} \sigma: \nabla^{\boldsymbol{x}} \eta \mathrm{d} V\right] \cdot \delta \boldsymbol{u} & =D\left[\int_{\mathcal{B}_{0}} \boldsymbol{P}: \nabla^{X} \eta \mathrm{d} V\right] \cdot \delta \boldsymbol{u} \\
& =\int_{\mathcal{B}} \frac{1}{J} \boldsymbol{A}:\left(\nabla^{\boldsymbol{x}} \delta \boldsymbol{u} \boldsymbol{F}\right):\left(\nabla^{\boldsymbol{x}} \boldsymbol{\eta} \boldsymbol{F}\right) \mathrm{d} V \\
& =\int_{\mathcal{B}} \boldsymbol{a}:\left(\nabla^{x} \delta \boldsymbol{u}\right):\left(\nabla^{\boldsymbol{x}} \boldsymbol{\eta}\right) \mathrm{d} V
\end{aligned}
$$




$$
\begin{aligned}
D\left[\int_{\Gamma} \boldsymbol{\eta} \cdot \boldsymbol{\sigma} \cdot \boldsymbol{n d} A\right] \cdot \delta \boldsymbol{u} & =D\left[\int_{\Gamma_{0}} \boldsymbol{\eta} \cdot \boldsymbol{P} \cdot \boldsymbol{N \mathrm { d } A}\right] \cdot \delta \boldsymbol{u} \\
& =\int_{\Gamma_{0}} \boldsymbol{\eta} \cdot \boldsymbol{A}: \nabla^{\boldsymbol{X}} \delta \boldsymbol{u} \cdot \boldsymbol{N \mathrm { d } A} \\
& =\int_{\Gamma} \frac{1}{\boldsymbol{J}} \boldsymbol{\eta} \cdot \boldsymbol{A}:\left(\nabla^{\boldsymbol{x}} \delta \boldsymbol{u} \boldsymbol{F}\right) \cdot \boldsymbol{F}^{T} \cdot \boldsymbol{n} \mathrm{d} A \\
& =\int_{\Gamma} \boldsymbol{\eta} \cdot \boldsymbol{a}: \nabla^{\boldsymbol{x}} \delta \boldsymbol{u} \cdot \boldsymbol{n} \mathrm{d} A
\end{aligned}
$$

where $\boldsymbol{a}$ is defined in (35).

\section{Verification of the local nonlocal damage model}

We here adopt a uniaxial loading example to verify the nonlocal damage model. As shown in Fig. 18, a bar with a length of $10 \mathrm{~m}$ is fixed on the left bound and subjected to an incremental displacement on the right bound. The material is described by an isotropic elastic damage model. The elastic modulus is $10 \mathrm{GPa}$, Poisson's ratio is 0.2 , and $\varepsilon_{0}$ and $\varepsilon_{f}$ in (30) are $1 \times 10^{-4}$ and $2 \times 10^{-3}$, respectively. We set the size of element as $h=0.5,1$ and $2 \mathrm{~m}$. The internal length for the integral regularization is $2.2 \mathrm{~m}$. Figure 19 plots the curves of $\varepsilon-\sigma$ for different mesh sizes and constitutive models. We can see that the results are mesh-dependent for local damage model and less dissipation energy for the finer grid. On the contrary, the results are identical for different meshes with the nonlocal damage model. We further compare the distribution of

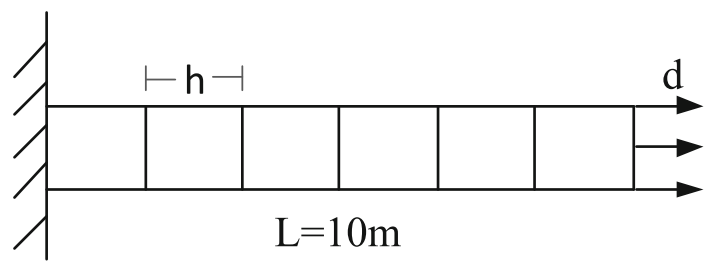

Fig. 18 Model of a bar under a uniaxial loading

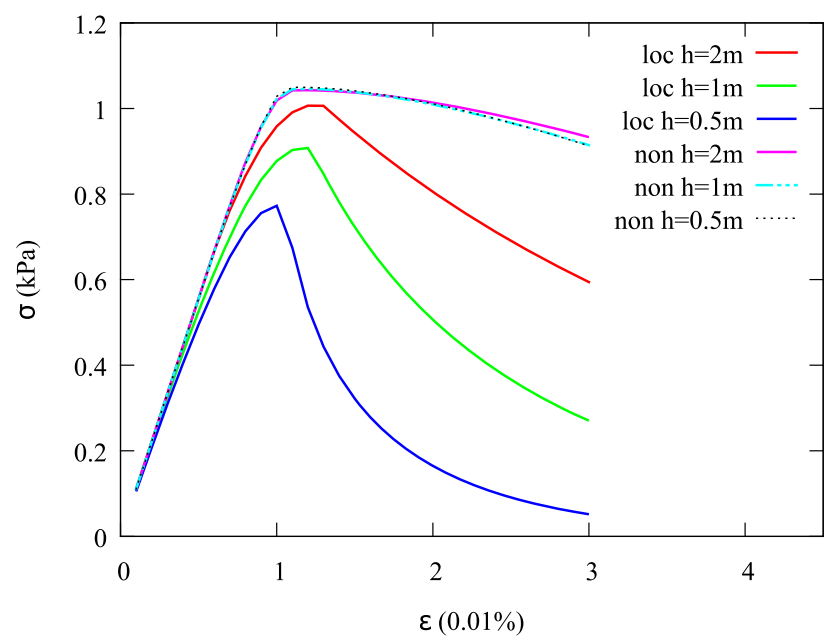

Fig. 19 Stress-strain curves with different meshes for local (loc) and nonlocal damage (non) models strain along the horizontal axis and find that the distribution is almost uniform for the nonlocal damage model and the strain localizes at the rightmost element for the local damage model. We thus believe our implementation of the nonlocal damage model is correct.

\section{References}

1. Abe K, Soga K, Bandara S (2013) Material point method for coupled hydromechanical problems. J Geotech Geoenviron Eng 140(3):04013033

2. Andersen S, Andersen L (2010) Modelling of landslides with the material-point method. Comput Geosci 14(1):137-147

3. Bardenhagen SG, Brackbill JU, Sulsky D (2000) The materialpoint method for granular materials. Comput Methods Appl Mech Eng 187(3-4):529-541

4. Belytschko T, Liu WK, Moran B, Elkhodary K (2013) Nonlinear Finite Elements for Continua and Structures. Wiley, London

5. Charlton TJ, Coombs WM, Augarde CE (2017) iGIMP: an implicit generalised interpolation material point method for large deformations. Comput Struct 190:108-125

6. Chen J-S, Pan C, Roque CMOL, Wang H-P (1998) A lagrangian reproducing kernel particle method for metal forming analysis. Comput Mech 22(3):289-307

7. Chen J-S, Hillman M, Chi S-W (2017) Meshfree methods: progress made after 20 years. J Eng Mech 143(4):04017001

8. Cortis M, Coombs W, Augarde C, Brown M, Brennan A, Robinson S (2018) Imposition of essential boundary conditions in the material point method. Int J Numer Methods Eng 113(1):130-152

9. Cuitino A, Ortiz M (1992) A material-independent method for extending stress update algorithms from small-strain plasticity to finite plasticity with multiplicative kinematics. Eng Comput 9(4):437-451

10. de Borst R, Crisfield MA, Remmers JJC, Verhoosel CV et al (2012) Non-linear finite element analysis of solids and structures. Wiley, London

11. de Souza Neto EA, Peric D, Owen DRJ (2011a) Computational methods for plasticity: theory and applications. Wiley, London

12. de Souza Neto EA, Peric D, Owen DRJ (2011b) Computational methods for plasticity: theory and applications. Wiley, London

13. Fernández-Méndez S, Huerta A (2004) Imposing essential boundary conditions in mesh-free methods. Comput Methods Appl Mech Eng 193(12-14):1257-1275

14. Gong M (2015) Improving the material point method. Ph.D. dissertation, University of New Mexico

15. Guan PC, Chen JS, Wu Y, Teng H, Gaidos J, Hofstetter K, Alsaleh M (2009) Semi-lagrangian reproducing kernel formulation and application to modeling earth moving operations. Mech Mater 41(6):670-683

16. Guan PC, Chi SW, Chen JS, Slawson TR, Roth MJ (2011) Semilagrangian reproducing kernel particle method for fragment-impact problems. Int J Impact Eng 38(12):1033-1047

17. Guilkey JE, Weiss JA (2003) Implicit time integration for the material point method: quantitative and algorithmic comparisons with the finite element method. Int J Numer Methods Eng 57(9):13231338

18. Gupta R, Ando E, Salager S, Wang K, Sun WC (2018a) Open source database for validating and falsifying discrete mechanics models using synthetic granular materials-Part I: experimental tests with particles manufactured by a 3D printer

19. Gupta R, Salager S, Wang K, Sun WC (2018b) Open-source support toward validating and falsifying discrete mechanics models using synthetic granular materials-Part I: experimental tests with particles manufactured by a 3D printer. Acta Geotechn, pp 1-15 
20. Jirásek M (2007) Nonlocal damage mechanics. Revue européenne de génie civil 11(7-8):993-1021

21. Kimmel R, Shaked D, Kiryati N, Bruckstein AM (1995) Skeletonization via distance maps and level sets. Comput Vis Image Underst 62(3):382-391

22. Kuhn MR, Sun WC, Wang Q (2015) Stress-induced anisotropy in granular materials: fabric, stiffness, and permeability. Acta Geotech 10(4):399-419

23. Kularathna S, Soga K (2017) Implicit formulation of material point method for analysis of incompressible materials. Comput Methods Appl Mech Eng 313:673-686

24. Li S, Liu WK (2002) Meshfree and particle methods and their applications. Appl Mech Rev 55(1):1-34

25. Liu C, Sun Q, Yang Y (2017) Multi-scale modelling of granular pile collapse by using material point method and discrete element method. Procedia Eng 175:29-35

26. Liu Y, Sun WC, Fish J (2016a) Determining material parameters for critical state plasticity models based on multilevel extended digital database. J Appl Mech 83(1):011003

27. Liu Y, Sun WC, Yuan Z, Fish J (2016b) A nonlocal multiscale discrete-continuum model for predicting mechanical behavior of granular materials. Int J Numer Methods Eng 106(2):129-160

28. Lopes A, Brodlie K (2003) Improving the robustness and accuracy of the marching cubes algorithm for isosurfacing. IEEE Trans Vis Comput Gr 9(1):16-29

29. Main A, Scovazzi G (2018a) The shifted boundary method for embedded domain computations. Part I: poisson and stokes problems. J Comput Phys 372:972-995

30. Main A, Scovazzi G (2018b) The shifted boundary method for embedded domain computations. Part II: linear advectiondiffusion and incompressible Navier-Stokes equations. J Comput Phys

31. Mazars J (1984) Application de la mécanique de l'endommagement au comportement non linéaire et à la rupture du béton de structure. THESE DE DOCTEUR ES SCIENCES PRESENTEE A L'UNIVERSITE PIERRE ET MARIE CURIE-PARIS 6

32. Mota A, Sun WC, Ostien JT, Foulk JW, Long KN (2013) Liegroup interpolation and variational recovery for internal variables. Comput Mech 52(6):1281-1299

33. Mueller R, Gross D, Maugin GA (2004) Use of material forces in adaptive finite element methods. Comput Mech 33(6):421-434

34. Na S, Sun W (2017) Computational thermo-hydro-mechanics for multiphase freezing and thawing porous media in the finite deformation range. Comput Methods Appl Mech Eng 318:667-700

35. Nair A, Roy S (2012) Implicit time integration in the generalized interpolation material point method for finite deformation hyperelasticity. Mech Adv Mater Struct 19(6):465-473

36. Nitsche J (1971) Über ein variationsprinzip zur lösung von dirichlet-problemen bei verwendung von teilräumen, die keinen randbedingungen unterworfen sind. Abhandlungen aus dem Mathematischen Seminar der Universität Hamburg 36(1):9-15. https:// doi.org/10.1007/BF02995904 ISSN 1865-8784

37. Ortiz M (2002) Course notes for computational solid mechanics

38. Papadrakakis M (1981) A method for the automatic evaluation of the dynamic relaxation parameters. Comput Methods Appl Mech Eng 25(1):35-48

39. Qinami A, Bryant EC, Sun WC, Kaliske M (2019) Circumventing mesh bias by $\mathrm{r}$ - and h-adaptive techniques for variational eigenfracture. Int J Fract

40. Schillinger D, Ruess M (2015) The finite cell method: a review in the context of higher-order structural analysis of cad and imagebased geometric models. Arch Comput Methods Eng 22(3):391455

41. Sirjani A, Cross GR (1991) On representation of a shape's skeleton. Pattern Recognit Lett 12(3):149-154
42. Song T, Main A, Scovazzi G, Ricchiuto M (2018) The shifted boundary method for hyperbolic systems: embedded domain computations of linear waves and shallow water flows. J Comput Phys 369:45-79

43. Sulsky D, Kaul A (2004) Implicit dynamics in the material-point method. Comput Methods Appl Mech Eng 193(12-14):1137-1170

44. Sulsky D, Gong M (2016) Improving the material-point method. In: Innovative numerical approaches for multi-field and multi-scale problems. Springer, Berlin, 217-240

45. Sulsky D, Chen Z, Schreyer HL (1994) A particle method for history-dependent materials. Comput Methods Appl Mech Eng 118(1-2):179-196

46. Sulsky D, Zhou S-J, Schreyer HL (1995) Application of a particlein-cell method to solid mechanics. Comput Phys Commun 87(12):236-252

47. Sulsky D, Schreyer H, Peterson K, Kwok Ron, Coon M (2007) Using the material-point method to model sea ice dynamics. J Geophys Res: Oceans 112(C2)

48. Sun W (2015) A stabilized finite element formulation for monolithic thermo-hydro-mechanical simulations at finite strain. Int $\mathbf{J}$ Numer Methods Eng 103(11):798-839

49. Sun W, Andrade JE, Rudnicki JW (2011a) Multiscale method for characterization of porous microstructures and their impact on macroscopic effective permeability. Int J Numer Methods Eng 88(12): 1260-1279

50. Sun W, Andrade JE, Rudnicki JW, Eichhubl P (2011b) Connecting microstructural attributes and permeability from 3D tomographic images of in situ shear-enhanced compaction bands using multiscale computations. Geophys Res Lett 38(10)

51. Sun WC, Wong T (2018) Prediction of permeability and formation factor of sandstone with hybrid lattice boltzmann/finite element simulation on microtomographic images. Int J Rock Mech Min Sci 106:269-277

52. Sun WC, Kuhn MR, Rudnicki JW (2013a) A multiscale DEMLBM analysis on permeability evolutions inside a dilatant shear band. Acta Geotechnica 8(5):465-480

53. Sun WC, Ostien JT, Salinger AG (2013b) A stabilized assumed deformation gradient finite element formulation for strongly coupled poromechanical simulations at finite strain. Int J Numer Anal Methods Geomech 37(16):2755-2788

54. Truster TJ, Nassif O (2017) Variational projection methods for gradient crystal plasticity using lie algebras. Int J Numer Methods Eng 110(4):303-332

55. Wang B, Vardon PJ, Hicks MA, Chen Z (2016) Development of an implicit material point method for geotechnical applications. Comput Geotech 71:159-167

56. Wang K, Sun W (2016) A semi-implicit discrete-continuum coupling method for porous media based on the effective stress principle at finite strain. Comput Methods Appl Mech Eng 304:546-583

57. Wang K, Sun WC (2018) A multiscale multi-permeability poroplasticity model linked by recursive homogenizations and deep learning. Comput Methods Appl Mech Eng 334:337-380

58. Wang K, Sun WC (2019) Meta-modeling game for deriving theory-consistent, microstructure-based traction-separation laws via deep reinforcement learning. Comput Methods Appl Mech Eng 346:216-241

59. Wriggers P (2008) Nonlinear finite element methods. Springer, Berlin

60. Zienkiewicz OC, Zhu JZ (1992) The superconvergent patch recovery (SPR) and adaptive finite element refinement. Comput Methods Appl Mech Eng 101(1-3):207-224

Publisher's Note Springer Nature remains neutral with regard to jurisdictional claims in published maps and institutional affiliations. 